\title{
Uranus Deep Atmosphere Revealed
}

\author{
IMKE DE PATER, ${ }^{*}, 1$ PAUL N. ROMANI, ${ }^{\dagger}, 2$ AND SUSHIL K. ATREYA $\ddagger$ \\ *Astronomy Department, Campbell Hall 601, University of California, Berkeley, California 94720; \\ $\dagger$ National Aeronautics and Space Administration, Goddard Space Flight Center, code 693.2, \\ Greenbelt, Maryland 20771; and $\ddagger$ Department of Atmospheric, Oceanic and Space Sciences, \\ University of Michigan, Ann Arbor, Michigan 48109
}

Received October 3, 1988; revised April 24, 1989

We have examined Uranus' radio spectrum and the latitudinal variation in its radio brightness temperature. We conclude that Uranus' spectrum cannot be explained with models based upon thermochemical equilibrium. The spectrum can only be matched if there is a low ammonia volume mixing ratio (a few times $10^{-7}$ between roughly $150<$ $T<240 \mathrm{~K}$ ), distributed uniformly over a large range of altitude. To remove sufficient $\mathrm{NH}_{3}$ at deep tropospheric levels through the formation of an $\mathrm{NH}_{4} \mathrm{SH}$-solid cloud, a large concentration of $\mathrm{H}_{2} \mathrm{~S}$ gas is needed, which implies that sulfur must be enhanced relative to its solar value by a factor of a few hundred. We further can constrain the $S / N$ ratio to be at least five times the solar value if the $\mathrm{H}_{2} \mathrm{O}$ volume mixing ratio is $<100 \times$ solar and equal to or larger than $3 \times$ solar if $\mathrm{H}_{2} \mathrm{O}$ is $\geq 500 \times$ solar. The elemental ratios derived from our work support the theory of planetary accretion by Pollack and Bodenheimer (1989, in Origin and Evolution of Atmospheres (S. Atreya, Ed.)). The constant mixing ratio of $\mathrm{NH}_{3}$ in the $145<T \leq 240 \mathrm{~K}$ range implies either vertical mixing on time scales much shorter than expected from reasonable values of the eddy diffusion coefficient, or supersaturation of $\mathrm{NH}_{4} \mathrm{SH}$ at levels where $T \leqslant 240 \mathrm{~K}$. The pole-equator gradient in Uranus' radio brightness temperature implies a latitude-dependent variation in the ammonia mixing ratio, ranging from a few times $10^{-6}$ at altitudes where the temperature is $145<T<240 \mathrm{~K}$ in the equatorial region (latitudes $<30-40^{\circ}$ ), a few times $10^{-7}$ at $145<$ $T<220 \mathrm{~K}$ at midlatitudes, and $\sim 10^{-7}$ down to $280 \mathrm{~K}$ in the polar region. Whereas the values in the equator and midlatitudes can be explained by condensation theories, the polar value can only be explained by invoking strong downdrafts of dry air (gas from which most of the ammonia vapor has been removed by condensation). A comparison of images taken at 2- and 6-cm wavelength show a difference in the spatial distribution of the brightness temperature. In addition, images at both wavelengths show time variability in the zonal brightness distribution.

\section{INTRODUCTION}

Observations at radio wavelengths provide the only means at this time for probing the deep atmosphere-pressure greater than a few bars-of the giant planets. Radio spectra of all four major planets, Jupiter, Saturn, Uranus, and Neptune, have been

\footnotetext{
${ }^{1}$ Alfred P. Sloan Fellow.

2 NAS/NRC Resident Research Associate. Now with SSAI.
}

obtained in the past (e.g., de Pater and Massie 1985, and references therein). These spectra generally show an increase in brightness temperature with increasing wavelength beyond $1.3 \mathrm{~cm}$, due to the combined effect of a decrease in opacity at longer wavelengths and an increase in temperature at increasing depth in the planet. The spectra of the four planets have been compared extensively with model atmosphere calculations (e.g., de Pater and Massie 1985, and references therein). Such cal- 
culations were usually performed for a uniform (or sometimes layered) adiabatic atmosphere. Since most of the opacity at centimeter wavelengths is due to ammonia gas, the mixing ratio of this gas was changed from a pure solar value to one which best fit the data. It was found that the ammonia mixing ratio on Jupiter and Saturn was a factor of 3-5 below the solar value in the upper part of the atmosphere $(P<1-$ 1.3 bar), and a few times higher than the solar value at deeper levels ( $P>2-4$ bar). The ammonia concentration on Uranus and Neptune, however, seemed depleted by about 2 orders of magnitude in the $\sim 1$ - to 50-bar region compared to the solar value.

The decrease, or depletion, of ammonia gas on all outer planets has been attributed in the past to the formation of an ammonium hydrosulfide cloud layer (e.g., Gulkis et al. 1978, Lewis and Prinn 1980, de Pater 1986). For this process to be an effective sink for ammonia, the $\mathrm{H}_{2} \mathrm{~S}$ mixing ratio must be nearly equal to that of $\mathrm{NH}_{3}$ at the base of the $\mathrm{NH}_{4} \mathrm{SH}$ cloud, as one molecule of $\mathrm{H}_{2} \mathrm{~S}$ combines with one molecule of $\mathrm{NH}_{3}$ to make one molecule of $\mathrm{NH}_{4} \mathrm{SH}$. The solar $\mathrm{S} / \mathrm{N}$ ratio is $\approx 1: 4.5$, implying an $\mathrm{S} / \mathrm{N}$ ratio on the outer planets above the solar value. For Jupiter and Saturn, the $\mathrm{NH}_{3}$ mixing ratio is still larger than the $\mathrm{H}_{2} \mathrm{~S}$ mixing ratio, but on Uranus and Neptune the $\mathrm{H}_{2} \mathrm{~S}$ mixing ratio must be nearly equal to or larger than the $\mathrm{NH}_{3}$ mixing ratio. For the latter planets, an alternative suggestion was made by Atreya and Romani (1985) and Fegley and Prinn (1986), who suggested a large loss of ammonia gas into an extensive aqueous solution cloud, requiring no selective $\mathrm{S} / \mathrm{N}$ enrichment from the solar ratio.

In addition, Uranus' radio spectrum has some unique features. Its spectrum is virtually flat at wavelengths longward of $6 \mathrm{~cm}$ (de Pater and Gulkis, 1988), requiring an additional source of opacity in Uranus' deep atmosphere, from either a large concentration of ammonia gas, water vapor, an ocean or solid surface, or a subadiabatic or isothermal thermal structure at those lev- els. Also, the disk-averaged radio brightness temperature of Uranus is time variable (e.g., Klein and Turegano 1978, Gulkis et al. 1983), and high-resolution images of the planet exhibit a large pole-to-equator gradient in the brightness temperature (e.g., Briggs and Andrew 1980, Jaffe et al. 1984, de Pater and Gulkis 1988). The latter two facts might be related to each other (Gulkis and de Pater 1984).

Are these oddities in Uranus' radio spectrum in any way related to Uranus' evolution (e.g., large inclination angle of the rotation axis; its small, if any, internal heat source; its composition)? To investigate this problem, we began a thorough study of the planet's radio spectrum by modeling the atmospheric temperature-pressure structure and the composition as it was modified by condensation. By assuming a composition of the planet's deep (mixed) troposphere and a level where both the temperature and pressure are known, it is possible to calculate the thermal structure and the locations and densities of the various cloud layers in the atmosphere by using hydrostatic and thermodynamic equilibrium. This modeling procedure has been applied to the outer planets by Lasker (1963), Lewis (1969), Weidenschilling and Lewis (1973), Atreya and Romani (1985), Romani (1986), Carlson et al. (1987), and Fegley and Prinn (1986). However, in none of these studies were the models used to generate a synthetic radio spectrum and compared to the actual planetary radio spectrum, as done here. This is a significant step, since only by doing so can the validity of the model be tested. The model used in this study is an updated and improved version of the one described extensively in Romani (1986).

\section{MODELS AND COMPUTATIONAL METHOD}

\section{A. Radio Spectrum}

Uranus' radio spectrum has been published in the past by several authors. We use the most recent publication by de Pater and Gulkis (1988), which contains all the 
published data of this planet taken prior to 1985. Several authors (e.g., Gulkis et al. 1983, Gulkis and de Pater 1984) discussed at length the observed time variability in Uranus' disk-averaged radio brightness temperature. The brightness temperature increased between 1965 and 1973, when Uranus' pole came into view. Radio images of the planet showed the pole to be warmer than the equator (Briggs and Andrew 1980, Jaffe et al. 1984, de Pater and Gulkis 1988). Gulkis and de Pater (1984) show that the increase in Uranus' disk-averaged radio brightness temperature can be explained to first order by the changing viewing aspect of the planet, assuming it has a pole-equator temperature gradient as displayed in the high-resolution radio images at $6 \mathrm{~cm}$. Since the objective in this paper is to explain the shape of Uranus' radio spectrum, we will concentrate on the data taken after 1973; these data are distinguished in all figures by filled circles, in contrast to crosses for data taken prior to 1973. The radio spectrum thus defined is characterized by a rather steep increase in brightness temperature between $3 \mathrm{~mm}$ and $6 \mathrm{~cm}$; longward of $6 \mathrm{~cm}$ the spectrum flattens out.

The disk-averaged brightness temperature at radio wavelengths, $T_{\mathrm{D}}$, is calculated by a numerical integration over optical depth, $\tau$, and position $\mu$ on the disk. The disk-averaged brightness, $B_{\nu}\left(T_{\mathrm{D}}\right)$, is given by

$$
B_{\nu}\left(T_{\mathrm{D}}\right)=2 \int_{0}^{1} \int_{0}^{\infty} B_{\nu}(T) \exp ((-\tau / \mu) d \tau / \mu d \mu .
$$

The brightness $B_{\nu}(T)$ is given by the Planck function, and optical depth $\tau_{\nu}(z)$ is the integral of the total absorption coefficient over the altitude range $z$ at frequency $\nu$. The parameter $\mu$ is the cosine of the angle between the line of sight and local vertical. We approximated the shape of Uranus by a sphere rather than that of an oblate disk, which may introduce small errors (an estimated $<1-2 \%)$. We use the radiative transfer code developed by de Pater and Massie (1985) to compute the brightness temperature of the planet at the different wavelengths. We may note here that the millimeter part of Uranus' spectrum is influenced by the collision-induced absorption of hydrogen as well as absorption by ammonia gas, while the opacity in the centimeter part of the spectrum is dominated by ammonia gas, water vapor, and droplets (de Pater and Massie 1985). We used a line shape for ammonia gas such as that suggested by de Pater and Massie (1985): Ben Reuven at centimeter wavelengths, and a modified Van Vleck Weiskopf line shape at millimeter wavelengths. This shape agrees with the recent work by Steffes and Jenkins (1987).

\section{B. Atmospheric Structure AND Composition}

The altitude distribution of the chemical species and the temperature-pressure profile were calculated using Romani's (1986) cloud model, which is briefly described here. The major difference between the model used in this study and previous ones (Romani 1986, Atreya and Romani 1985) is that previously more $\mathrm{NH}_{3}$ gas was lost into the solution cloud than at equilibrium. This has been changed, and the model is now a strict "equilibrium" model. The calculations start deep in the atmosphere, below the formation of any clouds, with a specified temperature, pressure, and composition of one mole of gas. The model then steps up in altitude from the base level and the new temperature is calculated, assuming a dry adiabatic lapse rate, and the new pressure by using hydrostatic equilibrium. The partial pressures of the trace gases in the atmosphere are then calculated by multiplying their respective mixing ratios by the atmospheric pressure. From the new temperature and empirical formulas the equilibrium saturation vapor pressures of the following possible condensates are calculated: aqueous ammonia solution of vari- 
able concentration, water ice, ammonia ice, hydrogen sulfide ice, ammonium hydrosulfide solid, methane ice, and argon ice. An ice cloud is assumed to form at the level where the calculated partial pressure of the constituent exceeds its saturation vapor pressure. For the aqueous ammonia solution cloud to form, the saturated vapor pressures of both ammonia and water above the solution of the test concentration must be lower than the respective ammonia and water partial pressures. This, however, only serves as a first step. We then search for an equilibrium concentration: a solution which has the same concentration as that which forms from setting the partial pressures of $\mathrm{NH}_{3}$ and $\mathrm{H}_{2} \mathrm{O}$ in the atmosphere equal to their saturated vapor pressure. Previously, Atreya and Romani (1985) had used a solution cloud that had the lowest possible concentration of $\mathrm{NH}_{3}$. With the low ammonia vapor pressure above such a solution, they dissolved much $\mathrm{NH}_{3}$ into the cloud. $\mathrm{H}_{2} \mathrm{~S}$ is dissolved into this cloud using an empirical expression from Leyko (1964). Then for both the ice clouds and solution cloud the atmospheric mixing ratios of the condensing species are reduced to their saturation values. Since the $\mathrm{NH}_{4} \mathrm{SH}$ cloud forms as a result of a reaction between $\mathrm{NH}_{3}$ and $\mathrm{H}_{2} \mathrm{~S}$ gases, the test for $\mathrm{NH}_{4} \mathrm{SH}$ cloud formation is that the equilibrium constant of the reaction is exceeded. Both $\mathrm{NH}_{3}$ and $\mathrm{H}_{2} \mathrm{~S}$ are reduced in equal molar quantities until the product of their atmospheric pressures equals the equilibrium constant. The model then steps up in altitude using now either the dry or the appropriate wet adiabat. As the trace gases are removed from the atmosphere by condensation, "dry" air (an $\mathrm{H}_{2}-\mathrm{He}$ mixture) is entrained into the parcel to ensure the mixing ratios add up to one. This cycle is repeated until the tropopause temperature is reached. We note that dry air was entrained rather than a mixture of gases, for computational ease. Entraining the proper mixture of gases, in particular of $\mathrm{CH}_{4}$ in addition to the $\mathrm{H}_{2}-\mathrm{He}$ mixture, may change the adiabat slightly.
Effects on the calculated microwave spectrum, however, are negligible.

At the model base level the gases $\mathrm{H}_{2} \mathrm{O}$, $\mathrm{CH}_{4}, \mathrm{NH}_{3}$, and $\mathrm{H}_{2} \mathrm{~S}$ are present in selected enrichments relative to solar, and the $\mathrm{He} /$ $\mathrm{H}_{2}$ ratio is set to the observed value on Uranus, 0.18 by volume (Conrath et al. 1987). We note here that our volume mixing ratios are relative to the total atmosphere, not just $\mathrm{H}_{2}$ alone, so they are also mole fractions. The $\mathrm{CH}_{4}$ mixing ratio was always held at 30 times to reproduce the observed $2 \%$ mixing ratio in the upper troposphere, before the $\mathrm{CH}_{4}$ ice cloud begins to form (Lindal et al. 1987). The temperature and pressure at the base level were chosen so the Voyager radio occultation temperature of $101 \mathrm{~K}$ at 2.3 bar (Lindal et al. 1987) was reproduced.

The empirical vapor pressure equations used in the model have been listed previously. The ices $\mathrm{H}_{2} \mathrm{O}, \mathrm{NH}_{3}$, and $\mathrm{H}_{2} \mathrm{~S}$ are in Atreya (1986), and $\mathrm{CH}_{4}$ ice is in Romani and Atreya (1988). The vapor pressures of ammonia and water above aqueous ammonia are modeled as outlined in Romani (1986); the vapor pressures are calculated from Clausius-Clapeyron equations with concentration-dependent coefficients. The coefficients were derived by fitting Wilson's (1925) data. They also reproduce other data at higher temperatures (Clifford and Hunter 1933). For $\mathrm{NH}_{4} \mathrm{SH}$ the equilibrium constant, $K_{\mathrm{p}}$, is given by

$$
\log _{10} K_{\mathrm{p}}=\frac{\Delta S}{2.303 R}-\frac{\Delta H}{2.303 R T},
$$

where $\Delta S$ and $\Delta H$ are the change in entropy and enthalpy per degree Kelvin, respectively; $R$ is the gas constant; and $T$ is the temperature. A good fit to data given in Table 1 of Magnusson (1907) is obtained by using

$$
\log _{10} K_{\mathrm{p}}=14.83-(4715 / T)
$$

The equation for the wet adiabatic lapse rate is given by (e.g., Atreya and Romani 1985) 


$$
\begin{aligned}
& \frac{d T}{d z}=\frac{-\bar{m}_{g}}{\bar{C}_{\mathrm{p}}}[1+ \\
& \frac{\left.\frac{1}{R T}\left(\sum_{k} L_{k} X_{k}+\frac{2\left(X_{\mathrm{H}_{2} \mathrm{~S}} \cdot X_{\mathrm{NH}_{3}}\right) L_{\mathrm{RX}}}{\left(X_{\mathrm{H}_{2} \mathrm{~S}}+X_{\mathrm{NH}_{3}}\right)}\right)\right]}{\left[1+\frac{1}{\bar{C}_{\mathrm{p}} T^{2}}\left(\sum_{k} \frac{L_{k}^{2} X_{k}}{R}\right.\right.} \\
& \left.\left.\quad+\frac{\left(X_{\mathrm{H}_{2} \mathrm{~S}} \cdot X_{\mathrm{NH}_{3}}\right)}{\left(X_{\mathrm{H}_{2} \mathrm{~s}}+X_{\mathrm{NH}_{3}}\right)} \cdot L_{\mathrm{RX}} \cdot 10,845\right)\right]
\end{aligned}
$$

In Eq. (3), $T$ is the temperature in degrees Kelvin, $d z$ is the height increment, $m$ is the atmospheric mean molecular weight, $\mathrm{g}$ is the gravitational acceleration, $R$ is the universal gas constant, $C_{\mathrm{p}}$ is the mean molar heat capacity of the atmosphere, $X_{k}$ is the number of moles of gas $k$ that condense, $L_{k}$ is the latent heat released on condensation, and $L_{\mathrm{RX}}$ is the heat of reaction for the formation of $\mathrm{NH}_{4} \mathrm{SH}$. The heat capacities for $\mathrm{H}_{2} \mathrm{O}, \mathrm{NH}_{3}, \mathrm{H}_{2} \mathrm{~S}$, and $\mathrm{CH}_{4}$ are from Castellan (1971), while the heat capacity for $\mathrm{He}$ is that of an ideal gas. The latent heats are tabulated in Atreya (1986).

The effect of ortho-para $\mathrm{H}_{2}$ equilibration on the lapse rate is taken into account by modifying the $C_{\mathrm{p}}$ of $\mathrm{H}_{2}$, and thus the atmospheric $C_{\mathrm{p}}$ (Massie and Hunten 1982). We used the $C_{\mathrm{p}}$ of normal $\mathrm{H}_{2}$, while we assumed the relative ortho-para hydrogen populations to be in equilibrium (Trafton's "intermediate" hydrogen, 1976).

In Eq. (3), note that the wet adiabatic lapse rate is simply the dry adiabatic lapse rate, $m g / C_{\mathrm{p}}$, modified by the latent heat release of condensation. In the model it is possible to specify a pure dry adiabat, a pure wet adiabat, or the dry plus some correction to the wet. The latter best describes a global average. However, the effect of changing the lapse rate from the pure wet to pure dry does not affect our results regarding the radio brightness spectrum. The radio brightness temperature depends upon the integral over temperature of the ammonia mixing ratio (opacity), while at thermodynamic equilibrium the ammonia mixing ratio is exponentially dependent upon the temperature. The choice of adiabat affects only the temperature-pressure at the base level to ensure reproduction of the $101 \mathrm{~K}$ at 2.3 bar as observed by the Voyager radio occultation experiment (Lindal et al. 1987). The base level for a wet adiabat is much colder than for a dry adiabat because the latent heat released on condensation warms the atmosphere considerably.

\section{RESULTS}

Based upon an oversimplified model of giant planet formation and evolution, all gaseous planets were formed from the solar nebula and did not lose any of their primordial material, so they are expected to have a composition similar to that of the Sun. Even though it is generally accepted by now that this model is, at best, a first-order approximation, it still is instructive to note any deviations in composition from that expected for a solar composition atmosphere. Since carbon is known to be enhanced on Uranus by about a factor of $\mathbf{3 0}$ relative to the solar value, ${ }^{3}$ we used the observed rather than solar value for $\mathrm{CH}_{4}$. This changes the adiabat slightly, and hence influences the calculated spectrum of the planet. This "solar" composition model is shown by the solid curve in Fig. 1. As noted before (e.g., Gulkis et al. 1978), brightness temperatures of Uranus are much warmer than indicated by this model. As shown by Gulkis et al. (1978) and de Pater and Massie (1985), good fits can be obtained by lowering the ammonia mixing ratio by about 2 orders of magnitude. The dashed curve shows the spectrum from de Pater and Massie (1985) for an atmosphere with an ammonia mixing ratio of $7.0 \times 10^{-7}$ and an atmosphere which became isothermal at $260 \mathrm{~K}$. The steep increase in the calculated spectrum between wavelengths of 1 and $6 \mathrm{~cm}$ is due to a constant, low ammonia mixing ratio in the altitude range corresponding to

\footnotetext{
${ }^{3}$ We use the solar mixing ratios: $\mathrm{CH}_{4} / \mathrm{H}_{2}, 8.35 \times$ $10^{-4} ; \mathrm{NH}_{3} / \mathrm{H}_{2}, 1.74 \times 10^{-4} ; \mathrm{H}_{2} \mathrm{O} / \mathrm{H}_{2}, 1.38 \times 10^{-3} ; \mathrm{H}_{2} \mathrm{~S} /$ $\mathrm{H}_{2}, 3.76 \times 10^{-5}$.
} 


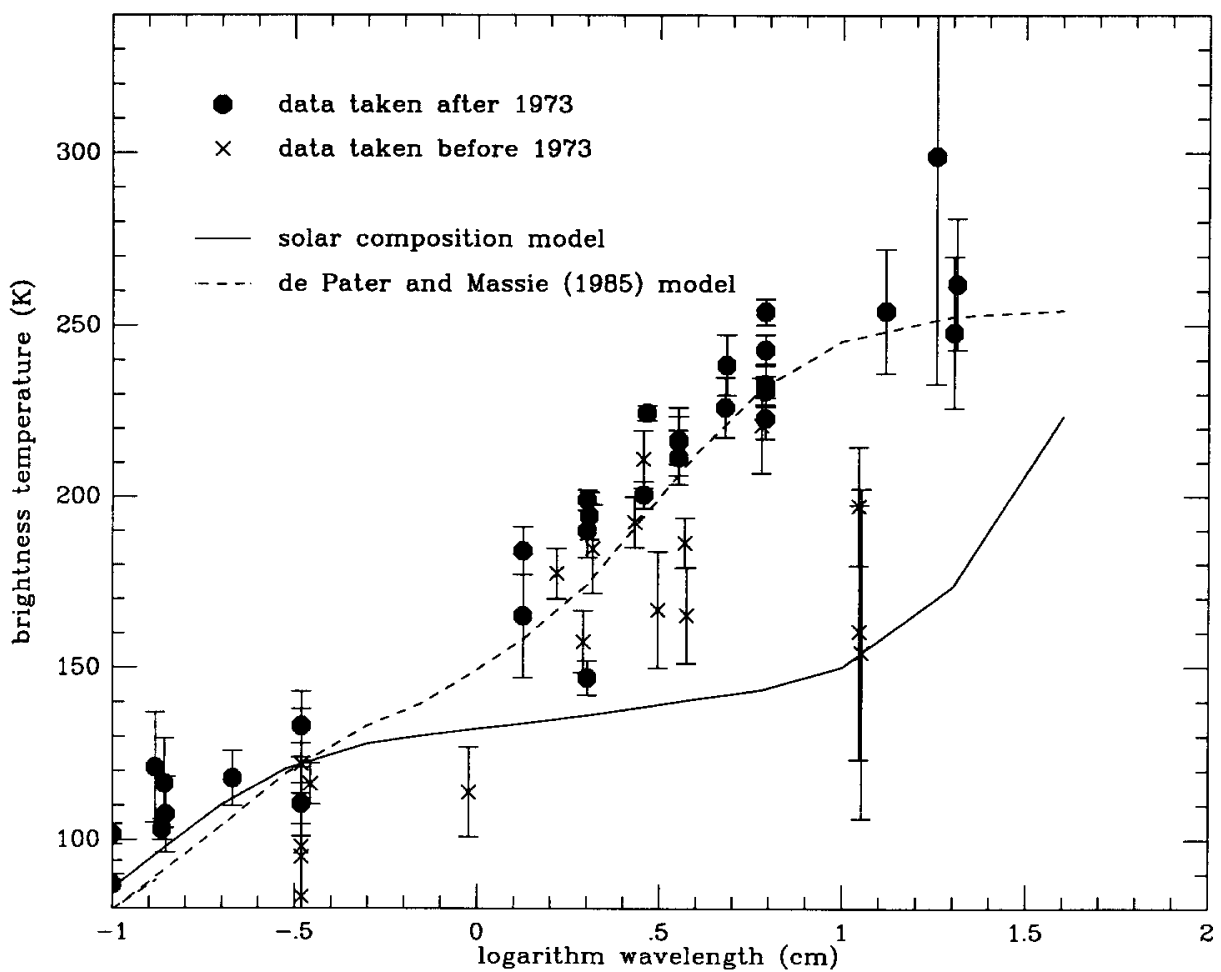

FIG. 1. Uranus' radio spectrum: filled circles are data points taken after 1973; crosses are points taken before 1973. - A model atmosphere calculation of a solar composition atmosphere (except carbon, which we always assumed as 30 times the solar value). ---, A model atmosphere calculation from de Pater and Massie (1985) with $90 \% \mathrm{H}_{2}, 8 \% \mathrm{He}, 2 \% \mathrm{CH}_{4}$, and mixing ratios of $10^{-6} \mathrm{H}_{2} \mathrm{O}$ and $7 \times$ $10^{-7} \mathrm{NH}_{3}$. The atmosphere was assumed to be isothermal at levels in the atmosphere below which the temperature was $260 \mathrm{~K}$.

temperatures of $\sim 160$ and $260 \mathrm{~K}$. The leveling off of the spectrum at wavelengths longward of $6 \mathrm{~cm}$ was enforced in this particular calculation by an isothermal atmosphere. Similar results can be obtained by assuming a large opacity, due to, e.g., an ammonia mixing ratio of the order of $1.0 \times$ $10^{-3}$ (i.e., at least 10 times the solar value) at levels in the atmosphere below $260 \mathrm{~K}$. The key problem to solve with our present calculations is how to account for the vertical structure (or absence thereof) in the ammonia mixing ratio, assuming that ammonia is not depleted in the deep atmosphere. In Section $3 \mathrm{~A}$ we present results obtained from thermochemical equilibrium models (as outlined in Section 2); in Section 3B we discuss disequilibrium models, and in Sec- tion $3 \mathrm{C}$ we address the question of a possible subsolar mixing ratio of $\mathrm{NH}_{3}$ throughout the entire atmosphere. We deduce the latitudinal variation in $\mathrm{NH}_{3}$ by fitting resolved images of Uranus' brightness temperature in Section 4. In Section 5A we summarize our findings on Uranus' composition; in Section 5B we compare the results regarding elemental mixing ratios to models of planetary formation, and in Section $5 \mathrm{C}$ we discuss the theories involved to explain our results on the vertical distribution of the atmospheric constituents.

\section{A. Chemical Equilibrium Models}

In this section we show results of a model in which all clouds are assumed to form in thermochemical equilibrium, as outlined in 


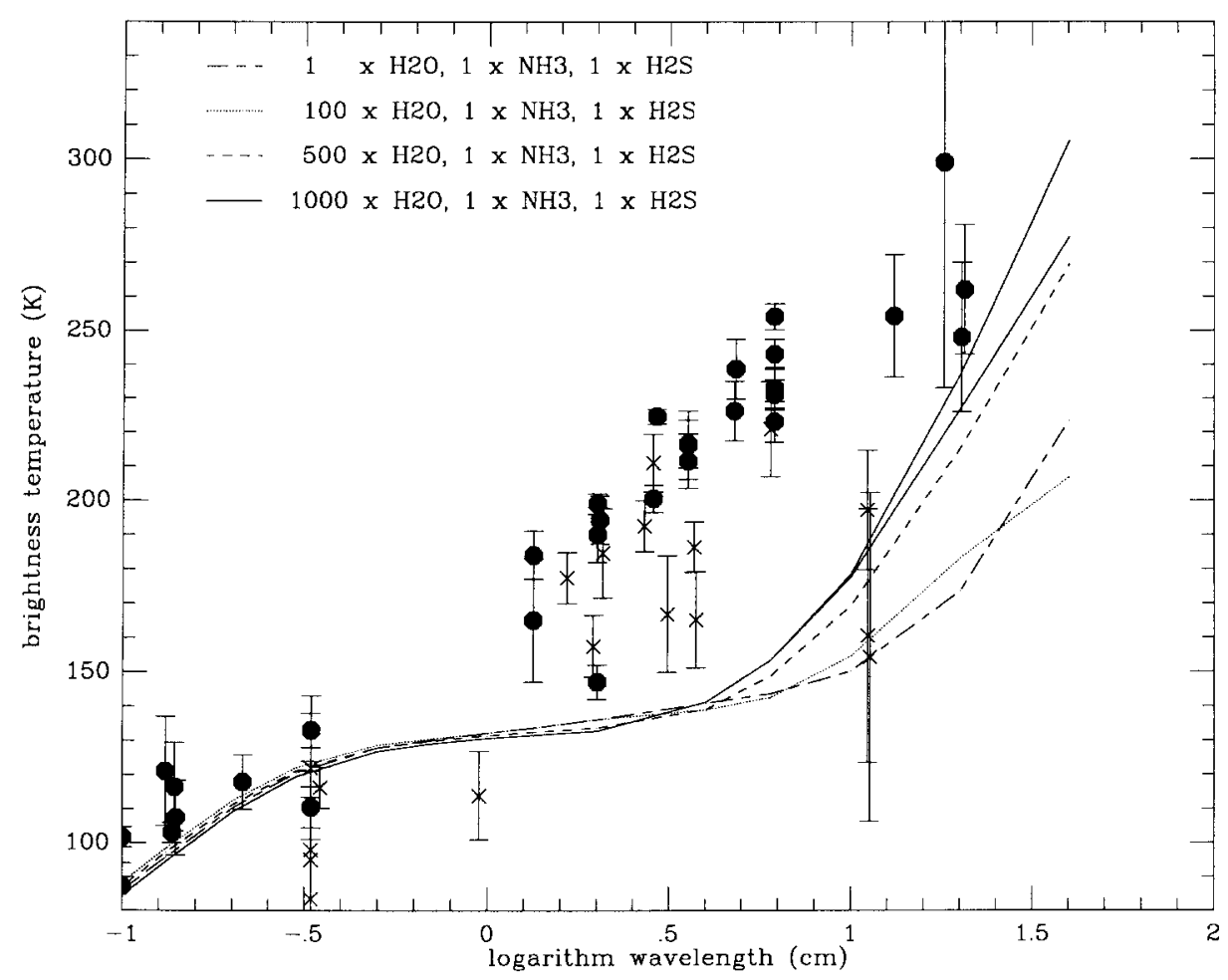

FIG. 2. Uranus' spectrum with superimposed four calculations for a model atmosphere in chemical equilibrium: solar mixing ratios for $\mathrm{NH}_{3}$ and $\mathrm{H}_{2} \mathrm{~S}$, while $\mathrm{H}_{2} \mathrm{O}$ is equal to $1 \times, 100 \times, 500 \times$ and $1000 \times$ the solar value. For the $1000 \times$ solar value we calculated the curve without (upper solid line) and with (lower solid line) inclusion of absorption by water droplets.

Section 2. Since the main source of opacity in Uranus' atmosphere at centimeter wavelengths is ammonia gas, we will show the effects on the radio spectrum due to ammonia loss from the atmosphere by cloud formation. Figure 2 shows the change in the microwave spectrum if the $\mathrm{H}_{2} \mathrm{O}$ mixing ratio in Uranus' atmosphere is increased from 1 to 100,500 , and 1000 times the solar value. For the $1000 \times \mathrm{H}_{2} \mathrm{O}$ case, we show the spectrum with (upper solid curve) and without (lower solid curve) absorption by water droplets; for the other calculations absorption by water droplets is always included. For a water mixing ratio $<100$ times the solar value, the solution cloud is too small to cause a noticeable change in the ammonia mixing ratio. In the $>500 \times$ $\mathrm{H}_{2} \mathrm{O}$ case, roughly $65 \%$ of the ammonia gas is dissolved in the solution cloud. Most of the decrease in the ammonia concentration ( $\sim 70 \%$ of the total ammonia loss) occurs over a small (few kilometers) altitude range at the bottom of the solution cloud. The reason for this is that for water mixing ratios of $>100 \times$ solar, the solution cloud forms as soon as the temperature drops below the critical temperature of water $(\sim 647$ $\mathrm{K})$. This results in a large first layer of the solution cloud into which a large fraction of the atmospheric $\mathrm{NH}_{3}$ dissolves. The difference between the cases with 500 and 1000 times the solar value is rather small. Even though over half the ammonia mixing ratio is dissolved into the solution cloud, we still need an additional sink $\left(\mathrm{NH}_{4} \mathrm{SH}\right.$-solid cloud) to reduce the ammonia mixing ratio to the observed value.

Figure 3a shows results of calculations from ammonia being removed by the forma- 

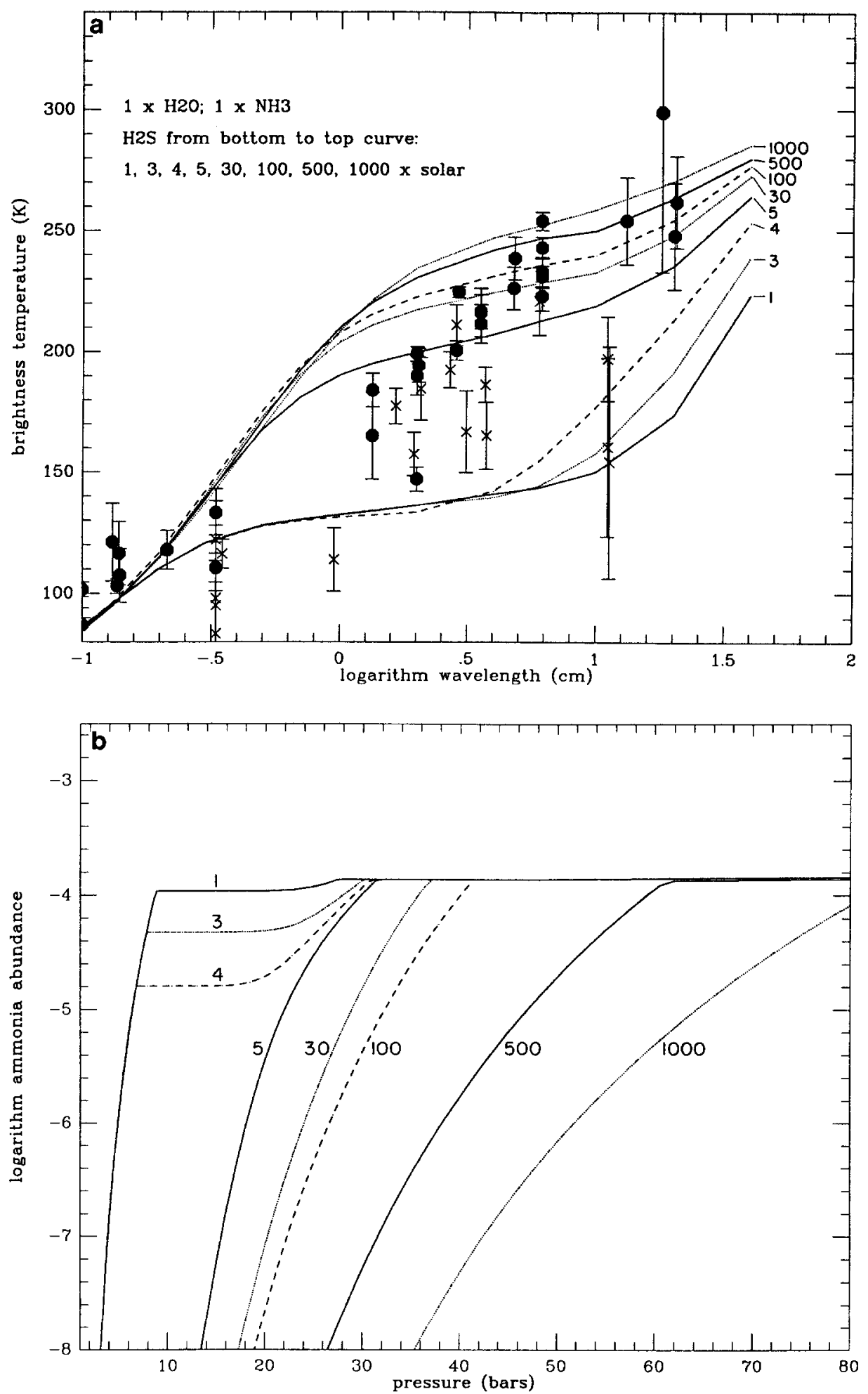

FIG. 3. (a) Calculations for a model atmosphere in chemical equilibrium: solar mixing ratios for $\mathbf{N H}_{3}$ and $\mathrm{H}_{2} \mathrm{O}$; the mixing ratio of $\mathrm{H}_{2} \mathrm{~S}$ with respect to the solar value varies from curve to curve as indicated: $1,3,4,5,30,100,500$, and 1000 . (b) The ammonia mixing ratio as a function of pressure for the same model atmospheres. 


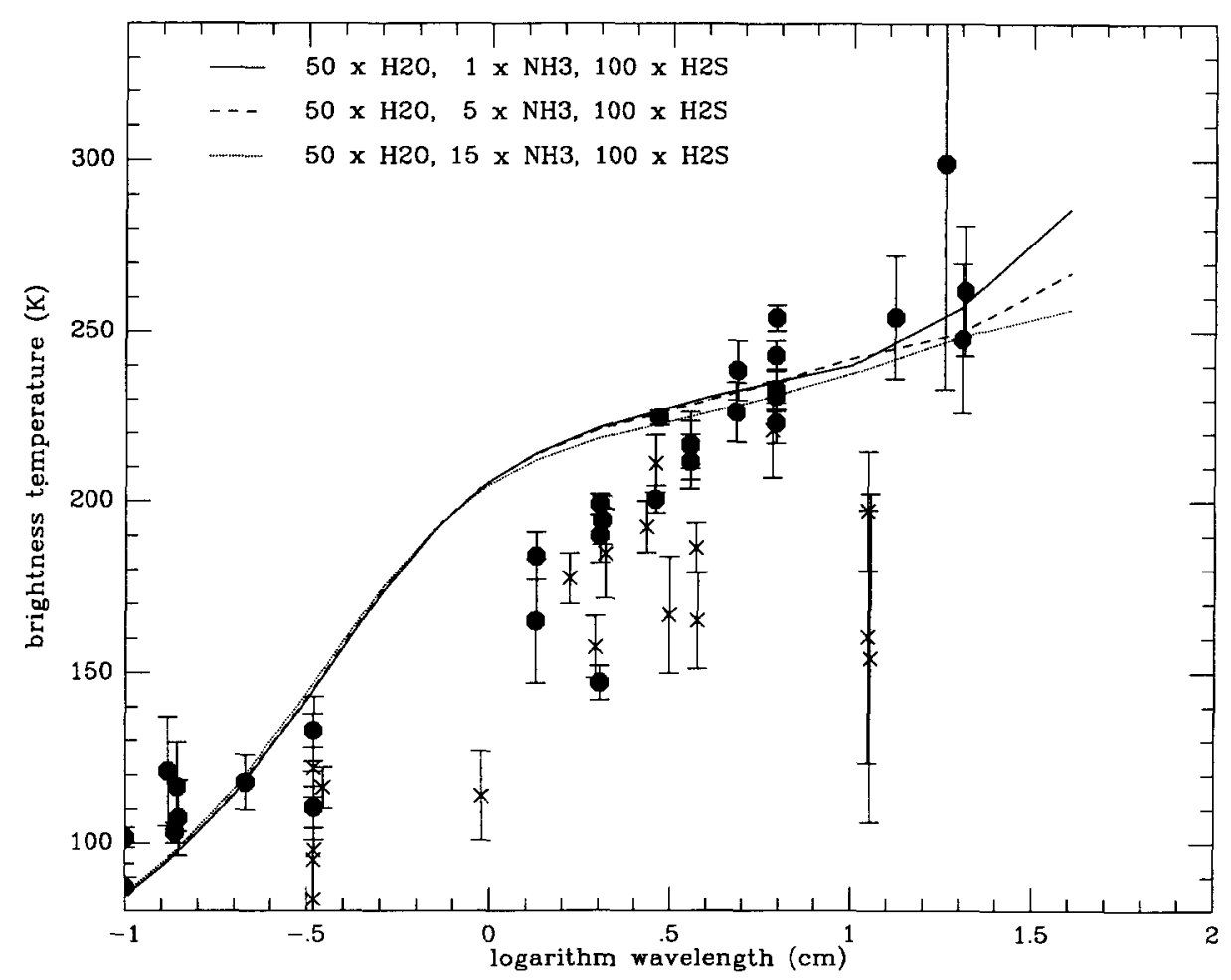

FIG. 4. Calculations for a model atmosphere in chemical equilibrium: the mixing ratios with respect to the solar value are $50 \times \mathrm{H}_{2} \mathrm{O}, 100 \times \mathrm{H}_{2} \mathrm{~S}$, and $1 \times, 5 \times$, and $15 \times \mathrm{NH}_{3}$ for the solid, dashed, and dotted lines, respectively.

tion of an $\mathrm{NH}_{4} \mathrm{SH}$-solid cloud. In these calculations we assumed solar mixing ratios for water and ammonia gas in Uranus' deep atmosphere. The $\mathrm{S} / \mathrm{N}$ ratio in the various calculations were taken equal to $1,3,4,5$, $30,100,500$, and 1000 times the solar value. As expected, ammonia gas is depleted significantly when the $\mathrm{H}_{2} \mathrm{~S}$ mixing ratio exceeds the $\mathrm{NH}_{3}$ mixing ratio, which happens at a $\mathrm{S} / \mathrm{N}$ between 4 and 5 . Figure $3 \mathrm{~b}$ shows the effect on the ammonia mixing ratio in a more direct way: the effect of the depletion is clearly visible, as is the location of the cloud base. The latter gets pushed to deeper levels if the $\mathrm{H}_{2} \mathrm{~S}$ mixing ratio and/or the $\mathrm{NH}_{3}$ mixing ratio is increased.

Although the average level of the spectra with $\mathrm{S} / \mathrm{N}$ ratios $>5$ agrees roughly with the data in the $1-$ to $10-\mathrm{cm}$ region (Fig. 3a), none of the spectra reproduces the observed shape. All calculated spectra ris steeply between 0.2 and $1.2 \mathrm{~cm}$, are rather flat up to $10 \mathrm{~cm}$, and show a gradual increase beyond $10 \mathrm{~cm}$. This general shape is due to the fact that in equilibrium models the ammonia mixing ratio is gradually decreased throughout the ammonium hydrosulfide cloud: the mixing ratio is large near the bottom of the cloud, a region probed at approximately $6-10 \mathrm{~cm}$, and practically zero near the top of the cloud layer, probed at the short-centimeter wavelengths. A change in the mixing ratios of various atmospheric constituents does not change the shape of the spectrum. An example is shown in Fig. 4: the three curves are model calculations with an enhancement of water with respect to the solar value by a factor of 50 , a mixing ratio of $\mathrm{H}_{2} \mathrm{~S}$ equal to 100 times the solar value, and $1 \times, 5 \times$, and $15 \times \mathrm{NH}_{3}$ shown by the solid, and curves, respectively. 
As shown by the dashed curve in Fig. 1, the shape of Uranus' spectrum can be matched only if the ammonia mixing ratio is equal to $\sim 7 \times 10^{-7}$ over the entire altitude range with temperatures between $\sim 160$ and 250-260 K.

\section{B. Disequilibrium Models}

We will discuss two scenarios which result in a uniform distribution of ammonia gas over a relatively large altitude range in the atmosphere. The first possibility is vertical mixing, the second is that the product of the partial pressures of $\mathrm{H}_{2} \mathrm{~S}$ and $\mathrm{NH}_{3}$ gas exceed the equilibrium constant, but without the condensation of $\mathrm{NH}_{4} \mathrm{SH}$ actually occurring (supersaturation). The likelihood of these processes will be addressed in Section 5 .

\section{i. Vertical Mixing}

An even distribution of ammonia gas over a large altitude range in the atmosphere can be obtained by vertical mixing. We suggest that upwelling and subsidence in Uranus' cloud layers could cause the clouds and gases to be redistributed "evenly" in altitude. This process, however, must be such as to conserve the total amount of molecules. Knowing that the best fit ammonia mixing ratio is about $0.5-$ $1.0 \times 10^{-6}$ over the altitude range probed at radio wavelengths (roughly $1-50$ bar), we calculated the range in altitude over which mixing must take place in order to produce a constant ammonia mixing ratio which best fits the observed spectrum. In fact, at short wavelengths the mixing ratio of $\mathrm{NH}_{3}$ gas which best fits the microwave spectrum depends critically on the altitude in the atmosphere below which mixing takes place. If all the mixing takes place at temperatures $T>160 \mathrm{~K}$, the average ammonia mixing ratio needs to be $7-9 \times 10^{-7}$ to give a reasonable fit to the observed spectrum; if the mixing is confined to deeper levels, the radio brightness temperature remains too high at short-centimeter wavelengths. If the mixing happens at $T>145 \mathrm{~K}$ rather than
$T>160 \mathrm{~K}$, the ammonia mixing ratio should be of the order $4-5 \times 10^{-7}$ to reproduce the observed spectrum at short wavelengths. At levels in the atmosphere where $T \lesssim 145 \mathrm{~K}$ ammonia gas will freeze out, which causes a drastic decrease in the gaseous ammonia mixing ratio. This is also required in models which match Uranus' microwave spectrum. Hence the region in which fast vertical mixing takes place is confined to levels in the atmosphere below $145-160 \mathrm{~K}$. The level in the atmosphere down to which this mixing extends is critical for the determination of the average ammonia mixing ratio. The average ammonia mixing ratio is roughly $1 / 10$ the mixing ratio at the bottom of this mixing region. The $\mathrm{NH}_{3}$ mixing ratio here depends most critically upon the ammonia mixing ratio in the deep atmosphere, and the altitude at which the $\mathrm{NH}_{4} \mathrm{SH}$ layer forms. If the mixing ratio of either $\mathrm{H}_{2} \mathrm{~S}$ and/or $\mathrm{NH}_{3}$ is increased, the formation of $\mathrm{NH}_{4} \mathrm{SH}$ starts at deeper levels in the atmosphere (see, e.g., Fig. 3b). If the water mixing ratio is increased, more ammonia and hydrogen sulfide dissolve into it, causing the altitude of the $\mathrm{NH}_{4} \mathrm{SH}$ formation to be raised in the atmosphere.

From Section 3A, Fig. 3, we conclude that the $\mathrm{S} / \mathrm{N}$ ratio must be at least five times the solar value to get a disk-averaged brightness temperature close to the observed values. The ammonia mixing ratio, averaged over an altitude range between 160 and $214 \mathrm{~K}$ is $9.3 \times 10^{-7}$, for a model atmosphere with solar mixing ratios for $\mathrm{H}_{2} \mathrm{O}$ and $\mathrm{NH}_{3}$ and five times the solar mixing ratio for $\mathrm{H}_{2} \mathrm{~S}$ (thus an $\mathrm{S} / \mathrm{N}$ ratio of 5; the spectrum based upon the equilibrium calculations was shown in Fig. 3, curve 5). The resulting spectrum for the vertically mixed atmosphere is shown by the dotted line in Fig. 5. Note that mixing in the atmosphere will influence all gases equally. The effect of the water vapor on the microwave spectrum will be minor since its averaged mixing ratio will be at most 1 order of magnitude larger than the $\mathrm{NH}_{3}$ mixing ratio and the absorption coefficient for water is a fac- 


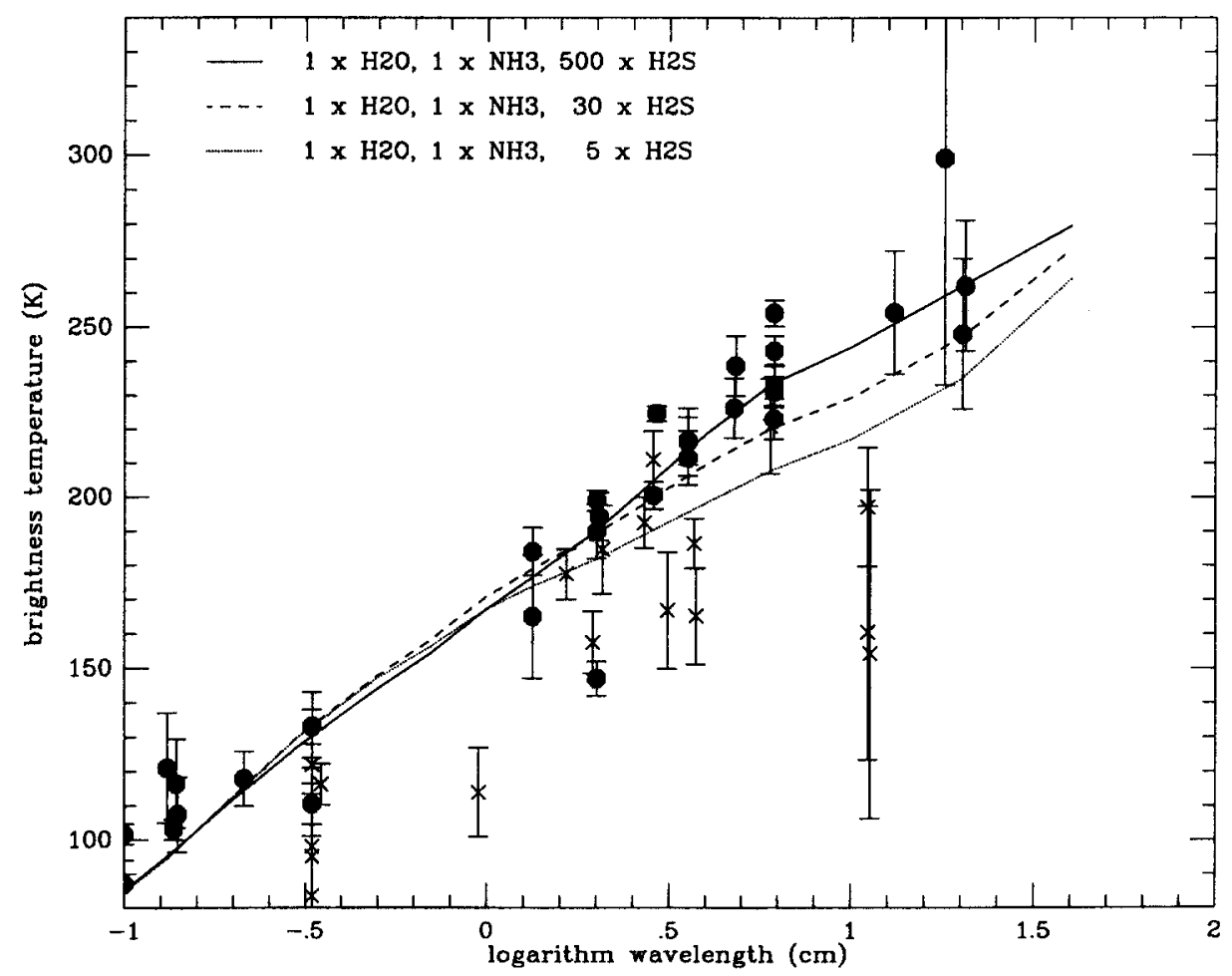

Fig. 5. Calculations for a model atmosphere in which vertical mixing is responsible for a uniform distribution of ammonia gas over a large range of altitudes. For all three calculations we have solar mixing ratios for $\mathrm{H}_{2} \mathrm{O}$ and $\mathrm{NH}_{3}$; the $\mathrm{H}_{2} \mathrm{~S}$ mixing ratio is $5 \times, 30 \times$, and $500 \times$ the solar value for the dotted, dashed, and solid line, respectively.

tor of 100 smaller than for ammonia (e.g., Goodman 1969). Even though the spectrum shown by the dotted line in Fig. 5 fits the short-wavelength data fairly well, the curve falls well below the data points at the longer wavelengths. An increase in the $S / N$ ratio to 30 , but leaving the $\mathrm{H}_{2} \mathrm{O}$ and $\mathrm{NH}_{3}$ mixing ratios equal to the solar value, results in the spectrum shown by the dashed curve. The fit is improved, but the curve still falls below the data points longward of $\sim 4 \mathrm{~cm}$. A mixing ratio of a few hundred times the solar value in $\mathrm{H}_{2} \mathrm{~S}$ is needed to push the formation of the $\mathrm{NH}_{4} \mathrm{SH}$ cloud down to a level such that $\mathrm{NH}_{3}$ gets depleted over large enough an altitude range to give a good fit to the microwave spectrum. The solid line in Fig. 5 is a calculation for $500 \times \mathrm{H}_{2} \mathrm{~S}, 1 \times$ $\mathrm{NH}_{3}, 1 \times \mathrm{H}_{2} \mathrm{O}$, averaged in altitude between 160 and $245 \mathrm{~K}$, where the $\mathrm{NH}_{3}$ mix- ing ratio is $6.8 \times 10^{-7}$. An increase in the $\mathrm{H}_{2} \mathrm{~S}$ mixing ratio to 1000 times the solar value does not change the latter profile noticeably.

In summary, Uranius' microwave spectrum can be reproduced quite well if the atmosphere is well mixed over a limited range in altitude: $\sim 150<T<\sim 250 \mathrm{~K}$. The mixing ratio of $\mathrm{H}_{2} \mathrm{~S}$ should be a few hundred times the solar $S$ value.

\section{ii. Supersaturation of $\mathrm{NH}_{4} \mathrm{SH}$}

As mentioned earlier (Section 2) the product of the partial pressures of $\mathrm{H}_{2} \mathrm{~S}$ and $\mathrm{NH}_{3}$ must exceed the equilibrium constant for the chemical reaction $\mathrm{H}_{2} \mathrm{~S}+\mathrm{NH}_{3} \rightarrow$ $\mathrm{NH}_{4} \mathrm{SH}$ (solid) to proceed. In this section we will determine the level in the atmosphere above which supersaturation must 


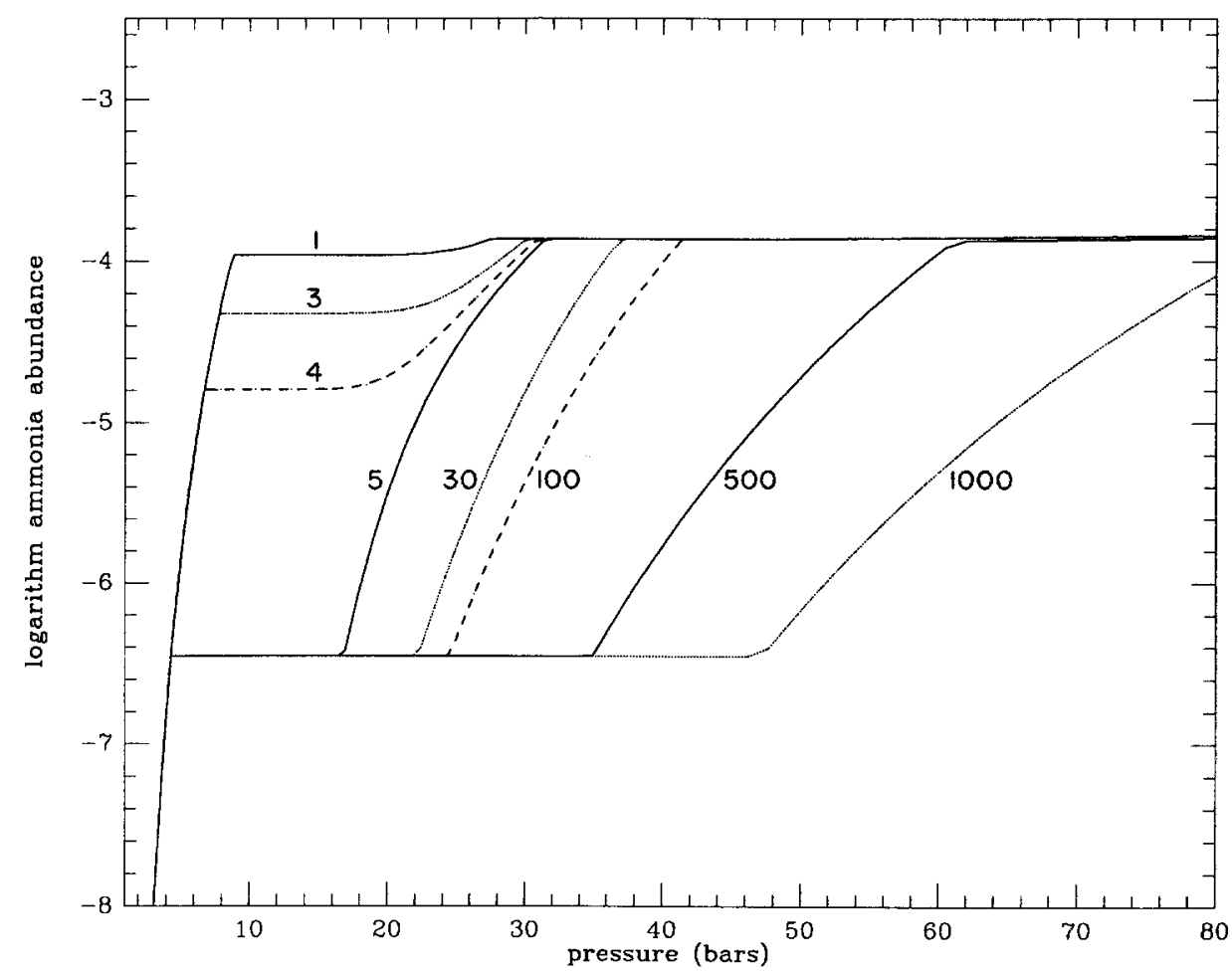

FIG. 6. The ammonia mixing ratio as a function of pressure in a model atmosphere in which ammonia gas is supersaturated above the $\mathrm{NH}_{4} \mathrm{SH}$ cloud.

take place in order to match the planet's radio spectrum.

When rising upward from deep within the atmosphere, the ammonia mixing ratio will decrease due to solution in the water cloud, formation of the ammonium hydrosulfide cloud, and, finally, the formation of an ammonia-ice cloud. The radio spectrum can be matched fairly well if we assume that the $\mathrm{NH}_{3}$ mixing ratio stays constant at 3-4 $\times$ $10^{-7}$ as soon as the concentration reaches this value in the $\mathrm{NH}_{4} \mathrm{SH}$ formation region. It will remain at this value until ammonia freezes out in the ice cloud. Hence, we change the ammonia mixing ratio curves such as those depicted in Fig. $3 b$ to those depicted in Fig. 6. The resulting microwave spectra for $\mathrm{S} / \mathrm{N}$ ratios of 5,30 , and 500 cannot be distinguished from those depicted in Fig. 5. Results for calculations in which we assumed larger mixing ratios for water and ammonia gas are also similar to those from calculations in which we assumed vertical mixing to redistribute the gases/clouds in the atmosphere (Section 3Bi). Hence, regardless of the redistribution process, $\mathrm{H}_{2} \mathrm{~S}$ needs to be enhanced by a few hundred above the solar value to fit Uranus' spectrum.

We note here that one would obtain the same type of results if the $\mathrm{H}_{2} \mathrm{~S}$ mixing ratio at the bottom of the $\mathrm{NH}_{4} \mathrm{SH}$ cloud is exactly $3-4 \times 10^{-7}$ less than the ammonia gas mixing ratio. The $\mathrm{NH}_{4} \mathrm{SH}$ cloud will then form until all of the $\mathrm{H}_{2} \mathrm{~S}$ has been used up.

If $\mathrm{H}_{2} \mathrm{~S}$ is enriched by a factor of 500 relative to the solar value, one would expect the other heavy elements to be enriched as well, although not necessarily by the same factor (see Section 5). An increase in the water mixing ratio by a factor of up to 1000 hardly influences the spectrum although the solubility of $\mathrm{NH}_{3}$ and $\mathrm{H}_{2} \mathrm{~S}$ gas into water increases. Profiles for a supersaturated at- 
mosphere with $500 \times \mathrm{H}_{2} \mathrm{~S}, 500 \times \mathrm{H}_{2} \mathrm{O}$, and an $\mathrm{NH}_{3}$ mixing ratio of $1,100,150$, and 200 times the solar value are shown in Fig. 7. From a comparison with the data, one can deduce that large concentrations of water absorb more $\mathrm{NH}_{3}$ gas than $\mathrm{H}_{2} \mathrm{~S}$ gas, such that the $\mathrm{S} / \mathrm{N}$ ratio can be decreased to 3 and still produce a good fit to Uranus' radio spectrum. Note further that no constraints on the ammonia mixing ratio other than the $\mathrm{S} / \mathrm{N}$ ratio can be determined (note subsolar values for ammonia gas are discussed in Section 3C). Similarly, we cannot determine the water mixing ratio, since this ratio is determined by the saturated vapor curve at the altitudes probed at radio wavelengths. If the water has a subsolar mixing ratio it is subsaturated, and Uranus' microwave spectrum will be changed significantly (see de Pater and Massie 1985). Since a subsolar value for the water mixing ratio in Uranus' atmosphere seems unlikely, we have not considered those cases.

\section{iii. Errors in the Equilibrium Constant?}

In addition to invoking large mixing ratios for chemical elements, we investigated the effect of varying the equilibrium constant, $K_{\mathrm{p}}$, for the reaction $\mathrm{NH}_{3}+\mathrm{H}_{2} \mathrm{~S} \rightarrow$ $\mathrm{NH}_{4} \mathrm{SH}$. Measurements of $K_{\mathrm{p}}$ date back to approximately the turn of the century (Isambert 1881, 1882, Walker and Lumsden 1897, Magnusson 1907, International Critical Tables 1930). In addition to these original sources Wagman et al. (1965) cite, without reference, values for the change in enthalpy, $\Delta H$, and in entropy, $\Delta S$, per degree Kelvin for $\mathrm{NH}_{4} \mathrm{SH}$ (see Eq. (2a)). With such ancient data we have some doubts about the accuracy of the measurements. Analysis of the above data produce very similar values for $K_{\mathrm{p}}$ with the exception of

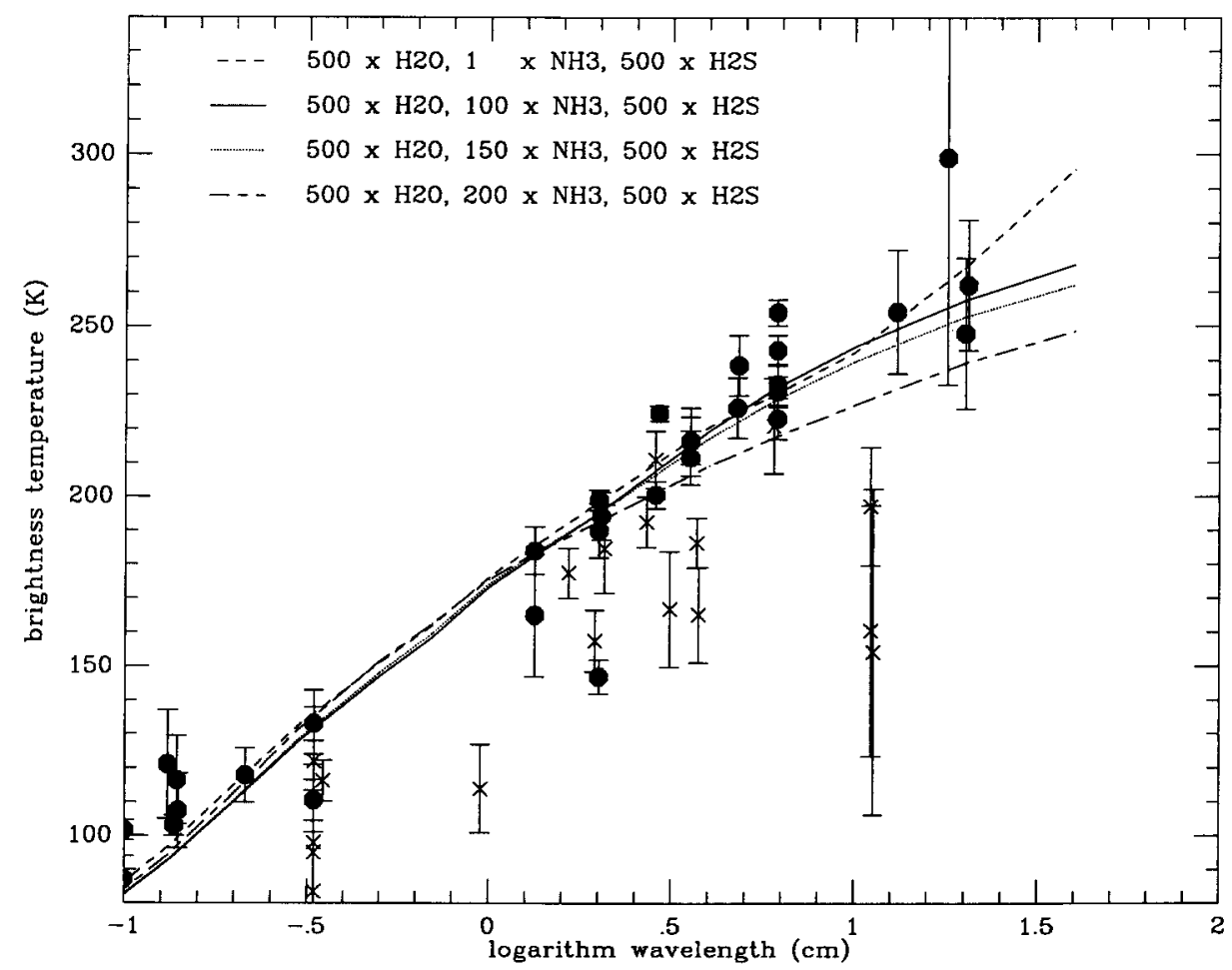

FIG. 7. Calculations for a model atmosphere in which ammonia is supersaturated: the mixing ratios of both $\mathrm{H}_{2} \mathrm{~S}$ and $\mathrm{H}_{2} \mathrm{O}$ are $500 \times$ solar; the mixing ratio of $\mathrm{NH}_{3}$ is $1 \times, 100 \times, 150 \times, 200 \times$ solar in the dashed, solid, dotted, and short-long dashed curves, respectively. 
Wagman et al. who produce a $K_{\mathrm{p}}$ an order of magnitude higher than the others, due to their high value for $\Delta S$. The entropy, $\Delta S$, typically varies by $\leqslant 6 \%$ among the numbers quoted by the different authors, while the enthalpy, $\Delta H$, typically agrees to within $3 \%$. A maximum reduction in $\Delta S, 2 \mathrm{cal} /$ mole/K, reduces $K_{\mathrm{p}}$ by a factor of 3 . We modeled several cases in which the equilibrium constant $K_{\mathrm{p}}$ was reduced by factors between 3 and 30 . This would enable a model less enriched in $\mathrm{NH}_{3}$ and $\mathrm{H}_{2} \mathrm{~S}$ to match the data as the $\mathrm{NH}_{4} \mathrm{SH}$ cloud layer would then form at a deeper level in the atmosphere. A calculation for $50 \times \mathrm{H}_{2} \mathrm{O}$, $100 \times \mathrm{H}_{2} \mathrm{~S}$, and $1 \times \mathrm{NH}_{3}$, with the equilibrium constant reduced by a factor of 10 gave results similar to those obtained for the enriched case of $500 \times \mathrm{H}_{2} \mathrm{~S}$ (curve 3 in Fig. 5). But this is outside the measured variation seen in the laboratory data.

In addition to the accuracy of the data, we are concerned about the extrapolation in temperature. All of the measurements were carried out in the temperature range from 273 to $298 \mathrm{~K}$, but the model runs from 660 to $50 \mathrm{~K}$. As $K_{\mathrm{p}}$ is exponentially dependent upon temperature, even small uncertainties in $\Delta H$ will have a strong effect upon the $\mathrm{NH}_{3}$ and $\mathrm{H}_{2} \mathrm{~S}$ mixing ratios. But as noted above most of the uncertainty in the measurements is in $\Delta S$ for the reaction. The other possible source of error in extrapolation would be a phase change in $\mathrm{NH}_{4} \mathrm{SH}$, but Bragin et al. (1977) did not see evidence for one down to $83 \mathrm{~K}$. In view of all of the above, we favor our calculations with the strongly enriched mixing ratios, reserving our doubts about $\boldsymbol{K}_{\mathrm{p}}$ until improved laboratory data become available.

\section{Subsolar $\mathrm{NH}_{3}$ Mixing Ratio THROUGHOUT ENTIRE ATMOSPHERE?}

So far we assumed that the ammonia mixing ratio is equal to or larger than the solar value in Uranus' deep atmosphere. However, we also need to investigate the possibility of a subsolar value throughout the entire atmosphere. Results of equilib- rium calculations are shown in Fig. 8. The solid line is a calculation for a supersaturation model with mixing ratios equal to $1 \times$ $\mathrm{H}_{2} \mathrm{O}, 1 / 10 \times \mathrm{NH}_{3}$ and $100 \times \mathrm{H}_{2} \mathrm{~S}$. Basically, as in the previous calculations, thermoequilibrium models show too high a temperature at short-centimeter wavelengths, and/or too low a value at $4-6 \mathrm{~cm}$; if the $\mathrm{H}_{2} \mathrm{~S}$ mixing ratio is raised to $\geqslant 100$ times the solar value, the brightness temperature at 4-6 $\mathrm{cm}$ can be matched. We again need the supersaturation (or vertical mixing) model to redistribute the ammonia gas uniformly over the relevant altitude range (solid line). The brightness temperature at $20 \mathrm{~cm}$, however, is rather high compared to the observed points, although the curve still touches the upper part of the error bars (regardless of the water mixing ratio). This suggests that the ammonia mixing ratio in Uranus' deep atmosphere is most likely larger than or equal to the solar value (note that we assumed the atmosphere to be adiabatic; see de Pater and Massie's calculation for the effect of an isothermal atmosphere, the dashed line in our Fig. 1).

\section{HIGH-RESOLUTION RADIO IMAGES}

From high-resolution radio observations it is well established by now that Uranus shows a large equator-pole gradient in brightness temperature (e.g., Briggs and Andrew 1980, Jaffe et al. 1984, de Pater and Gulkis 1988). De Pater and Gulkis (1988) show a few cross scans through such images, with some limb-darkening curves superimposed. We used their 2- and 6-cm images and fitted the observed center-tolimb scans. The results are shown in Figs. $9 a-9 d$, where the data are indicated by squares, and the model curves by the solid lines. The dashed line connects the points across Uranus disk over the pole, the dotted line is in the opposite direction. We did not attempt to take zonal averages, since that would force us to choose a point of symmetry on the disk (north pole, subsolar point??), and hence bias the data. However, note that the images are smeared in 


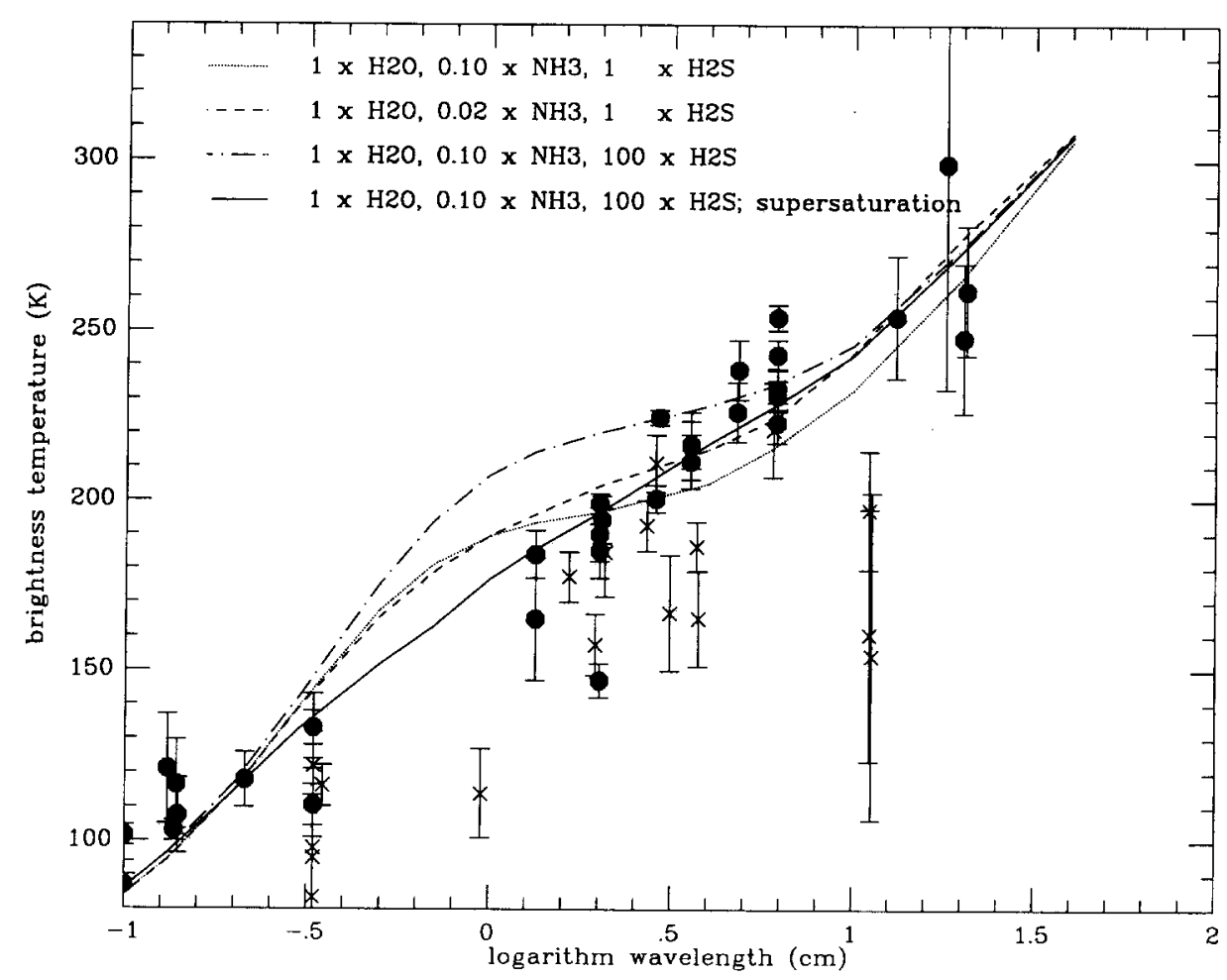

FIG. 8. Calculations for Uranus' atmosphere for subsolar $\mathrm{NH}_{3}$ mixing ratios: the precise values for the mixing ratios are shown on the figure. All curves in broken lines are for calculations in thermochemical equilibrium; the solid line is for a supersaturation model.

longitude due to the long integration times, so we cannot infer longitudinal asymmetries from the data.

The model curves in Fig. 9 are simple radio brightness temperature profiles; Fig. 10 shows the latitudinal variation in brightness temperature as required to fit the data. To separate the points on the curve, we added $2 \mathrm{~K}$ to all data from 1982 , and subtracted $2 \mathrm{~K}$ from all data in $1983 / 84$. This is within the range of uncertainty of our measurements. The solid circles and squares represent the brightness temperature at 6 and $2 \mathrm{~cm}$, respectively, in 1982, the crosses and triangles in 1983/84. The values are plotted as a function of "latitude" with respect to the point of symmetry (at $90^{\circ}$ ) on the planet. For the 6-cm data the temperature distribution was clearly symmetric about the south rotation pole, and the values along the $x$ axis in Fig. 10 represent the true planetocentric latitude for these data. At $2 \mathrm{~cm}$, no satisfactory fits could be obtained for a distribution which was symmetric about the pole, but only for a distribution which was symmetric about a point between the pole and the sub-earth (or subsolar) point. In 1982 the point of symmetry was at a sub-earth latitude of $82^{\circ}$ (the pole was at $\left.71.7^{\circ}\right)$; in 1984 it was at a sub-earth latitude of $84^{\circ}$ (the pole was at $81^{\circ}$ ). The model curves displayed in Fig. 9 are a convolution of the latitudinal radio brightness profile as plotted in Fig. 10, the limb-darkening curve, and beam convolution effect. The temperature structure near the equator is not well defined since the data are close to the limb of the planet. The absolute temperature scale was chosen such as to give a disk-averaged brightness temperature in agreement with Uranus' averaged spectrum: 183 and $191 \mathrm{~K}$ for the $2-\mathrm{cm}$ data in 

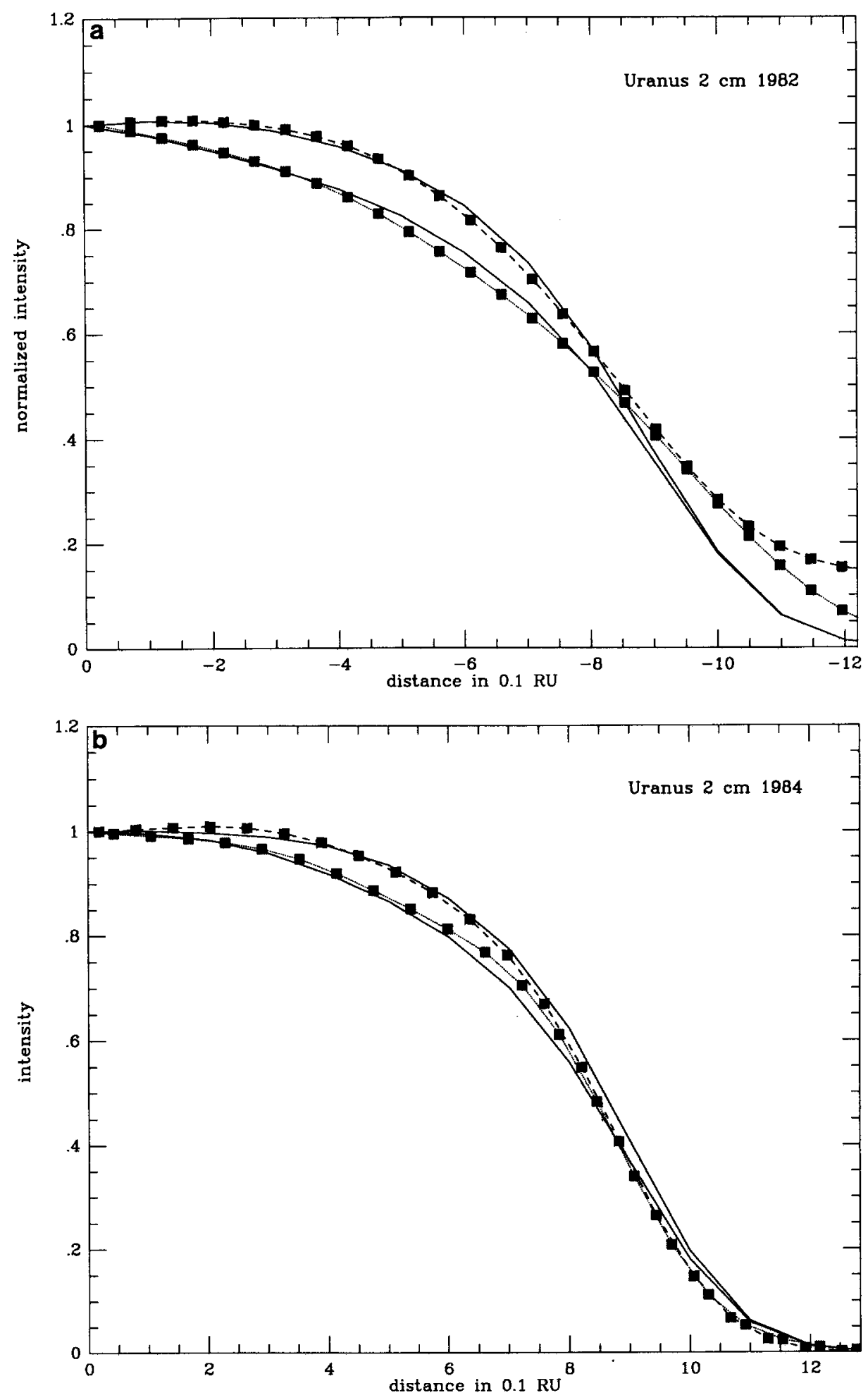

Fig. 9. Center-to-limb scans through high-resolution images of Uranus: the squares represent the data points. The points from Uranus' center over the pole are indicated by dotted lines, those in the opposite direction by dashed lines. The solid lines are scans in the same directions through a model disk, with a latitudinal radio brightness distribution as indicated in Fig. 10. The model temperatures were limb-darkened and convolved with the proper Gaussian beam to facilitate comparison with the data. 

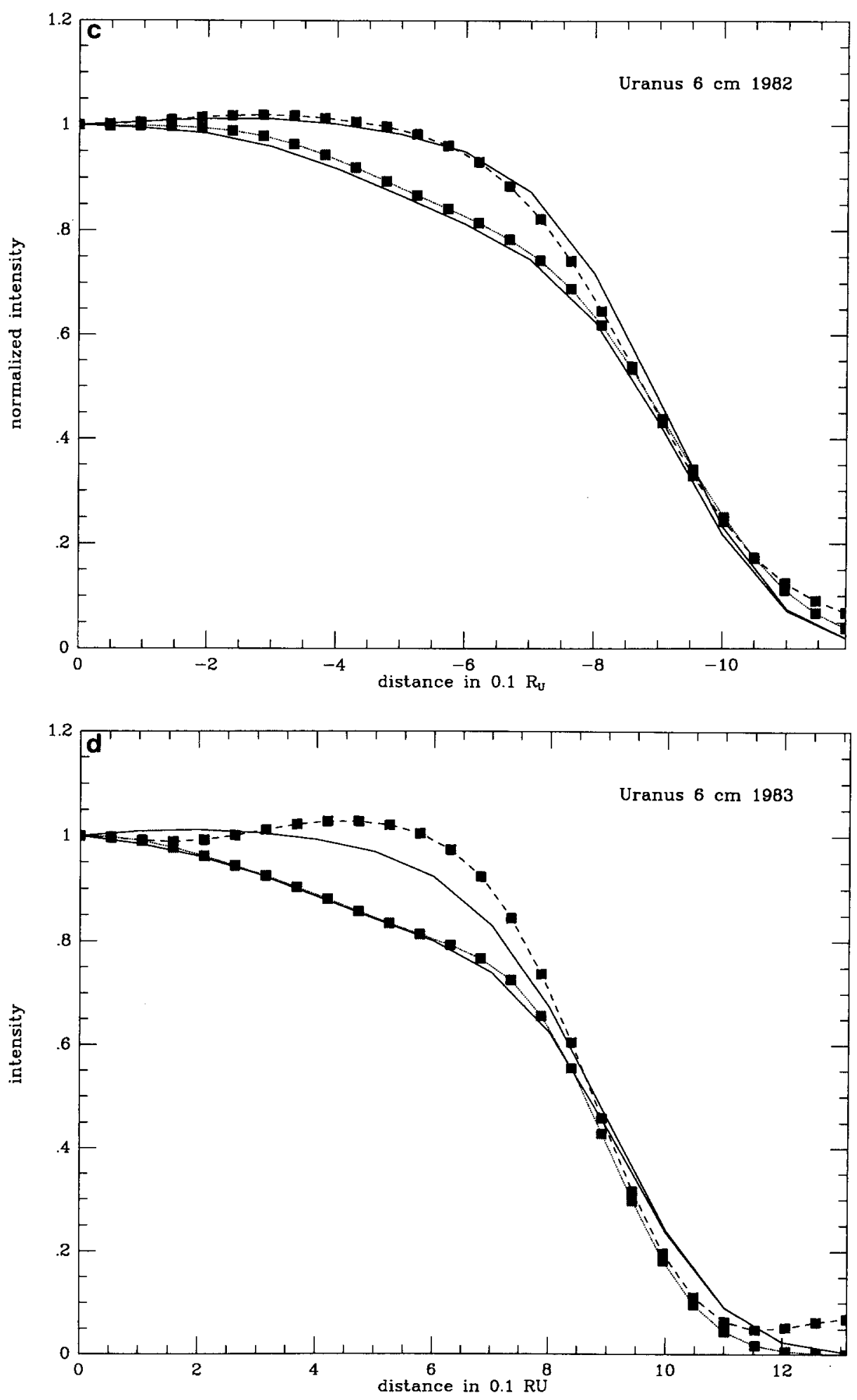

FIG. 9-Continued 


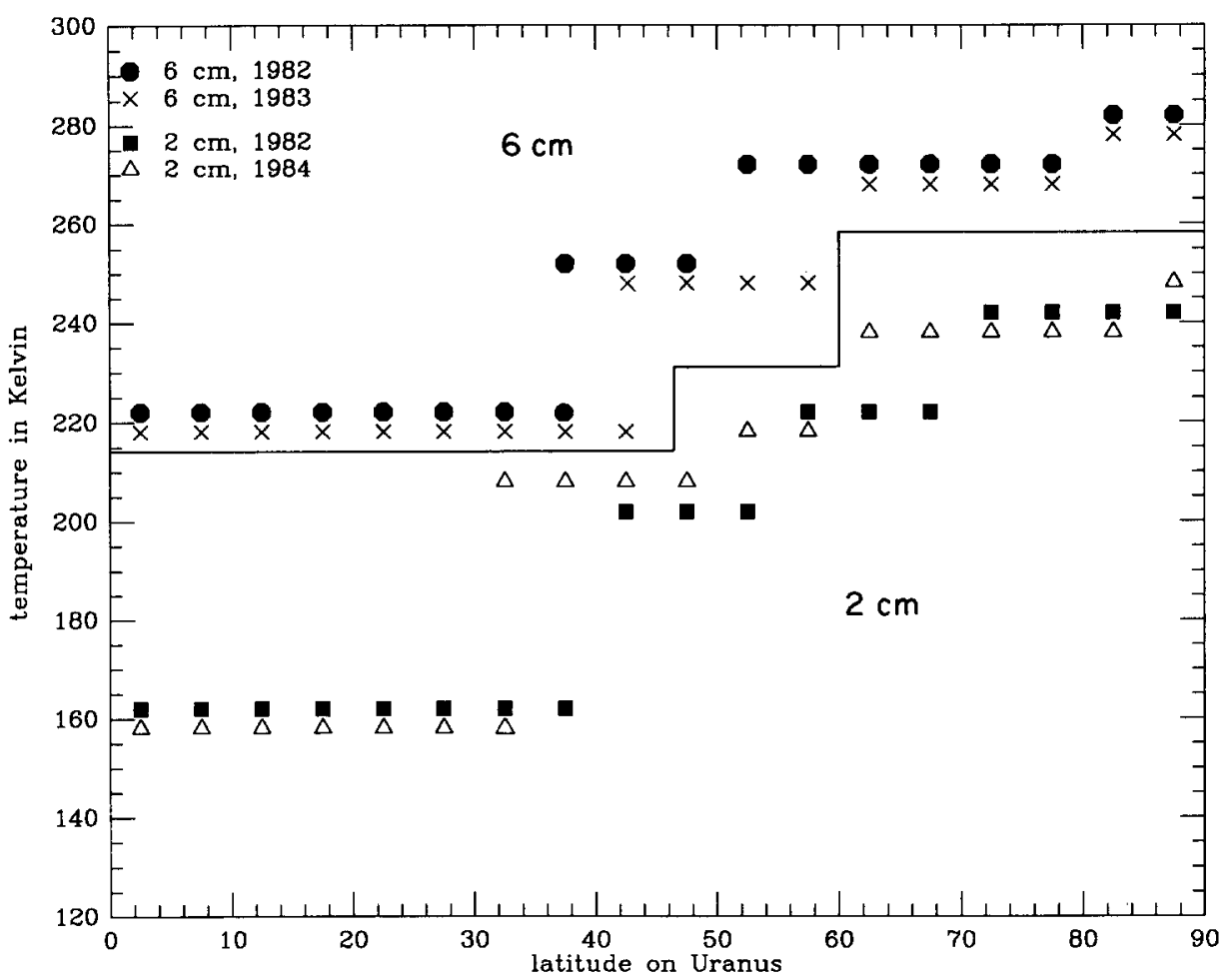

FIG. 10. The radio brightness temperature as a function of "latitude" with respect to the point of symmetry on the planet, for the best fit solutions shown in Fig. 9 (see text for further explanation).

1982 and 1984, respectively, and 234 and $231 \mathrm{~K}$ for the 6-cm data in both years, respectively.

The brightness temperature is distributed in bands on the planet, as expected for planets with zonal wind velocities. In a few cases the transition from one band to the next is ambiguous; in those cases we marked two points at the same latitude on the graph in Fig. 10. A comparison between the two 6-cm data sets clearly shows a change in the banded structure at latitudes between 50 and $60^{\circ}$ between the two years. The slope of the lower curve in Fig. 9d is much steeper than can be fitted with the temperature structure as measured in 1982. At $2 \mathrm{~cm}$, the temperature structure changed dramatically between 1982 and 1984 over the entire planet. In addition, the center of symmetry was not at the pole, but between the pole and the subsolar point. So besides the planet showing a banded or zonal structure at radio wavelengths, we can conclude from these observations that the structure changes dramatically with time. We may further note here that the 2- and 6-cm data from 1982 represent a time interval of only 1 month. The temperature structure, however, is quite different between the two wavelengths. Although most of this difference can be attributed to the fact that we probe different depths at the two wavelengths, a possibility exists that some of it might be due to short, temporal variations as well.

Despite the time variability in the spatial distribution of the radio brightness temperature, the general zonal distribution always seems present: a hot polar region at latitudes $>70^{\circ}: 270-280 \mathrm{~K}$ at $6 \mathrm{~cm}$ and $240-250$ $\mathrm{K}$ at $2 \mathrm{~cm}$; a cold equatorial band below roughly $30-40^{\circ}: 220 \mathrm{~K}$ at $6 \mathrm{~cm}$, and $160-170$ 
$\mathrm{K}$ at $2 \mathrm{~cm}$ (note that we cannot determine the temperature in the equatorial region, at latitudes below roughly $10^{\circ}$, due to the viewing geometry); and one or two bands at midlatitudes: $\sim 250 \mathrm{~K}$ at $6 \mathrm{~cm}$, and $200-220$ $\mathrm{K}$ at $2 \mathrm{~cm}$. In analogy with Jupiter (de Pater 1986), where the main source of opacity is ammonia gas, we attribute these temperature variations to latitudinal differences in the ammonia mixing ratio. However, we cannot rule out pole-equator variations in the temperature-pressure profiles.

As on Jupiter, the 2-cm brightness temperatures are most sensitive to the precise ammonia mixing ratio in the atmosphere, while the $6-\mathrm{cm}$ values are determined by a combination of the ammonia mixing ratio and the altitude range in the atmosphere over which the mixing ratio extends. For the polar regions we need an ammonia mixing ratio as low as $1-2.0 \times 10^{-7}$ between $\sim 150$ and $280 \mathrm{~K}$ to explain the $2-$ and $6-\mathrm{cm}$ temperatures. In the equatorial region, an ammonia mixing ratio of $-3.0 \times 10^{-6}$ is required between $\sim 150$ and $245 \mathrm{~K}$, while an mixing ratio of $3-7 \times 10^{-7}$ between $\sim 150$ and $250-260 \mathrm{~K}$ can explain the midlatitude values. In all these calculations the ammonia mixing ratio was assumed to vary in altitude according to the equilibrium model with $50-500 \times \mathrm{H}_{2} \mathrm{O}, 500 \times \mathrm{H}_{2} \mathrm{~S}$, and $15 \times$ $\mathrm{NH}_{3}$. We simply replaced the mixing ratio in the appropriate altitude range with the value mentioned (thus we did not try to conserve the total number of ammonia molecules). Hence, while the disk-averaged brightness temperature of Uranus as a function of wavelength can be matched fairly well with a cloud model containing a few hundred times the solar value of $\mathrm{H}_{2} \mathrm{~S}$, and in which the gases are mixed uniformly over an altitude range between $\sim 150$ and $250 \mathrm{~K}$, the latitudinal variation in temperature requires a slightly more elaborate cloud model. We need low mixing ratios to great depths in the polar regions, and higher, but still subsolar mixing ratios in the equatorial region. Thus, mixing is confined to different altitude regions at different latitudes on the planet.
In the context of the "supersaturation" model discussed in Section 3Bii), the 2- and 6- $\mathrm{cm}$ radio brightness temperatures in the equatorial region require supersaturation at $T<241 \mathrm{~K}$, with an ammonia mixing ratio of $2 \times 10^{-6}$; the midlatitude values can be roughly explained if supersaturation takes place at $T<222 \mathrm{~K}$, with an $\mathrm{NH}_{3}$ mixing ratio of $1-2 \times 10^{-7}$. The polar values, however, cannot be reproduced by invoking supersaturation above a certain altitude in the atmosphere. The ammonia mixing ratio is too low to too great depths in the atmosphere. This suggests downdrafts of dry air (gas from which ammonia gas is condensed out) in the polar regions.

We further note again that the brightness temperatures at $6 \mathrm{~cm}$ require symmetry around the pole, but at $2 \mathrm{~cm}$ around a point between the pole and the subsolar point. The images obtained in 1980 by Jaffe et al. (1984) show the point of symmetry at $6 \mathrm{~cm}$ also at the pole, but at the subsolar point at $2 \mathrm{~cm}$. Hence, the point of symmetry at $2 \mathrm{~cm}$ (upper atmosphere) appears to change with time, while the point of symmetry at $6 \mathrm{~cm}$ seems fixed at the pole. In addition, the zonal distribution of the brightness temperature at both wavelengths is changing with time, on time scales of weeks (?), months (?), or years.

One more remark on the apparent time variability of Uranus' atmosphere: the diskaveraged brightness temperature of the planet at $2 \mathrm{~cm}$ was only $147 \mathrm{~K}$ in $1980, \sim 40$ $\mathrm{K}$ below the mean value (Jaffe et al. 1984); if this value is correct, large (factors of $\sim 5$ ) global changes in the ammonia mixing ratio are required.

\section{DISCUSSION}

\section{A. Uranus' Composition}

It was shown in Section 3B that Uranus' spectrum can best be fitted if there is a low $\left(\sim 5 \times 10^{-7}\right)$ ammonia mixing ratio distributed uniformly over a large range in altitude $(\sim 150-240 \mathrm{~K})$. This hints at either a subsolar mixing ratio of ammonia gas throughout the entire atmosphere or the removal of 
$\mathrm{NH}_{3}$ gas at deep levels in Uranus' atmosphere. The first case was discussed in Section $3 \mathrm{C}$, the second scenario in Sections $3 \mathrm{~A}$ and $\mathrm{B}$. It appears that regardless of the value (sub-, or above solar) for $\mathrm{NH}_{3}$ gas, the $\mathrm{H}_{2} \mathrm{~S}$ mixing ratio needs to be large: $\mathrm{S}$ needs to be enhanced by a factor of a few hundred above the solar value, so that a cloud of $\mathrm{NH}_{4} \mathrm{SH}$ particles forms at really deep levels in the atmosphere. Vertical mixing, or supersaturation, of $\mathrm{NH}_{4} \mathrm{SH}$ would result in a uniform distribution of the gas over the altitude range as needed (see Section 5C).

\section{i. Regarding the Mixing Ratio of $\mathrm{NH}_{3}$}

In principle, $\mathrm{NH}_{3}$ could be present in a subsolar mixing ratio, if all or a substantial amount of the nitrogen in the protoplanetary nebula existed not as $\mathrm{NH}_{3}$, but in the form of $\mathrm{N}_{2}$, and $\mathrm{N}_{2} \rightarrow \mathrm{NH}_{3}$ conversion did not occur. In that case one would expect to see a rather large amount of $\mathrm{N}_{2}$ in the upper atmosphere, perhaps even larger than the amount of $\mathrm{NH}_{3}$. The upper limit to the detection of $\mathrm{N}_{2}$ by the Voyager UVS experiment is on the order of one part per billion at the 1-mbar level. This implies that most of the nitrogen in Uranus' atmosphere must be in the form of $\mathrm{NH}_{3}$, not $\mathrm{N}_{2}$. In addition, it was shown in Section $3 \mathrm{C}$ that a subsolar $\mathrm{NH}_{3}$ mixing ratio does not give quite the correct shape of Uranus' spectrum at long wavelengths, whereas calculations in which $\mathrm{NH}_{3}$ is enhanced compared to the solar value fit the spectrum quite well. Hence, a lower limit to the ammonia mixing ratio is the solar nitrogen mixing ratio. An upper limit can be derived from the minimum $\mathrm{S} / \mathrm{N}$ ratio required to lose sufficient ammonia gas through the formation of $\mathrm{NH}_{4} \mathrm{SH}$ particles to result in a good fit to the radio spectrum. A minimum $\mathrm{S} / \mathrm{N}$ ratio can be deduced from calculations severely enhanced in water content, since ammonia, and to a lesser extent $\mathrm{H}_{2} \mathrm{~S}$ gas, will partly dissolve into water. As mentioned in Section $3 \mathrm{~A}$, if water is enhanced by a factor of $>500$ above the solar $\mathrm{O}$ value, roughly $65 \%$ of the $\mathrm{NH}_{3}$ gas is dissolved into the cloud. From calcula- tions discussed in Section 3B we find that the minimum $\mathrm{S} / \mathrm{N}$ ratio is 3 . Hence an upper limit to $\mathrm{NH}_{3}$ would be $\sim 150 \times$ solar value, if $\mathrm{H}_{2} \mathrm{~S}$ is enhanced by a factor of $\sim 500$.

Unfortunately, no constraints on the water or $\mathrm{O}$ mixing ratio can be deduced from our data. We assumed the water mixing ratio to be at least equal to the solar value.

Our results should be compared with results published prior to this work by Carlson et al. (1987), and Fegley and Prinn (1986). The first authors suggested that the $\mathrm{H}_{2} \mathrm{~S}$ mixing ratio was exactly $10^{-6}$ less than the $\mathrm{NH}_{3}$ mixing ratio at the bottom of the $\mathrm{NH}_{4} \mathrm{SH}$ cloud layer. Hence, the $\mathrm{NH}_{4} \mathrm{SH}$ formation will take place until all the $\mathrm{H}_{2} \mathrm{~S}$ is depleted; the ammonia mixing ratio above the cloud is then $10^{-6}$. The result of such a process is similar to our results for a supersaturation model. In addition Carlson et al. suggested that slight latitude variations in the $\mathrm{H}_{2} \mathrm{~S}$ to $\mathrm{NH}_{3}$ ratio give rise to the observed latitude variations in the brightness temperature on Uranus with either $\mathrm{H}_{2} \mathrm{~S}$ or $\mathrm{NH}_{3}$ aloft above the $\mathrm{NH}_{4} \mathrm{SH}$ cloud. In their model they only considered low water mixing ratios and required a four- to fivefold enhancement of $\mathrm{S} / \mathrm{N}$ over solar $\mathrm{S} / \mathrm{N}$ to cause ammonia depletion. They did not place any limits on absolute mixing ratios of $\mathrm{H}_{2} \mathrm{~S}$ or $\mathrm{NH}_{3}$. The proposed mechanism for causing the $\mathrm{H}_{2} \mathrm{~S}$ to $\mathrm{NH}_{3}$ ratio to be nearly equal was photochemical conversion of $\mathrm{NH}_{3}$ to $\mathrm{N}_{2}$.

Our equilibrium model does agree with theirs, in that it requires a four to fivefold enhancement of $\mathrm{S} / \mathrm{N}$ from the solar ratio to deplete the atmosphere of ammonia for low water mixing ratios. However, at the condensation temperature of any $\mathrm{NH}_{3}$ above the $\mathrm{NH}_{4} \mathrm{SH}$ cloud (approximately at 145$150 \mathrm{~K})$, the Rayleigh scattering optical depth alone in the UV is on the order of 100. So photolysis is incapable of affecting the $\mathrm{NH}_{3}$ mixing ratios on present-day Uranus. Ammonia photolysis on Uranus requires a warmer Uranus ( $200 \mathrm{~K}$ at 1 bar) but not so warm that the $\mathrm{N}_{2}$ is then converted back to $\mathrm{NH}_{3}$. Since there is no com- 
pelling mechanism that would cause the $\mathrm{NH}_{3}$ and $\mathrm{H}_{2} \mathrm{~S}$ mixing ratios to be within a few times $10^{-7}$ of each other we reject such an "exact titration" model. Also, $\mathrm{NH}_{3}$ must be present at all latitudes above the $\mathrm{NH}_{4} \mathrm{SH}$ cloud to match the radio spectrum, not alternating between $\mathrm{H}_{2} \mathrm{~S}$ or $\mathrm{NH}_{3}$. It is important to keep in mind that these cloud models are essentially upwelling equilibrium models. A real planetary atmosphere is not likely to be in chemical equilibrium at all levels and will have regions of upwellings and downdrafts.

Fegley and Prinn (1986) consider atmospheric models all heavily enriched (1001000 ) in ice and rock-forming elements. They suggest that in such heavily enriched cases, most of the ammonia gas dissolves into the solution cloud. In fact, their ammonia mixing ratios are a few orders of magnitude below the "observed" values. They assume a higher solubility of ammonia gas into the water cloud than we did. If water is heavily enriched in Uranus' atmosphere $(>500 \times$ solar $)$, the minimum $\mathrm{S} / \mathrm{N}$ ratio required to fit the microwave spectrum using their vapor pressures is about 2 above the solar ratio.

\section{B. Comparison with Planetary FORMATION MODELS}

Prinn and Lewis (1973) were the first to suggest that, based upon cosmochemical arguments, the $\mathrm{S} / \mathrm{N}$ elemental ratio on Uranus might be enhanced above solar. When radio data on Uranus' spectrum became available, Prinn and Lewis' suggestion was revived and refined (e.g., Lewis and Prinn 1980, Pollack and Bodenheimer 1988).

Our findings on the $\mathrm{S} / \mathrm{N}$ elemental ratio, as well as the large mixing ratio of $S$, support models of Pollack and Bodenheimer (1989) for the formation and evolution of the outer planets and their atmospheres. These authors favor the "core-instability" model, in which the core of the giant planets is formed first by solid body accretion, similar to the formation of the Earth-like planets. When the mass reaches a critical value, gas accretion from the surrounding protoplanetary nebula becomes very rapid. This model accounts for the fact that the total mass made up of "heavy" elements (elements with atomic masses larger than $\mathrm{H}_{2}$ and $\mathrm{He}$ ) is similar for all four giant planets. This mass is roughly 5,25 , and 300 times larger for Jupiter, Saturn, and Uranus/Neptune, respectively, than would be expected from solar elemental mixing ratios. Furthermore, models of the interior structure of the planets show that the envelopes of the planets also contain large amounts of heavy elements. This can be accounted for in the core-instability hypothesis by the fact that late-accreting planetesimals have an increasing difficulty to penetrate through the denser and denser envelope.

Based upon the observed methane mixing ratios in the giant planets' atmospheres $(2.3,5.1,30-40$ times the solar value on Jupiter, Saturn, Uranus/Neptune, respectively), Pollack and Bodenheimer (1989, and references therein) estimate that about $10 \%$ of the carbon in the outer solar nebula was in the condensed phase during the epoch of planetary formation. Rocky and refractory elements, like $\mathrm{Mg}, \mathrm{Si}, \mathrm{P}, \mathrm{S}$, and water, were entirely in the solid phase, although part of the $\mathrm{O}$ was present in the form of mineral oxides and $\mathrm{CO}$ gas. The regular satellites of the giant planets are all made of rock and ice, with a mean rock/ice ratio of 55/45 by mass. However, since water is somewhat easier to dissolve in the envelopes of the forming giant planets, Pollack and Bodenheimer (1989) predict that the envelopes of the planets contain somewhat more water than rock by mass. So they expect the $\mathrm{O} / \mathrm{S}$ ratio to be slightly larger than the solar value. Like carbon, nitrogen is expected to have been present in both the gas (e.g., $\mathrm{N}_{2}$ ) and solid phases. Since the N/C ratio was found to be subsolar in meteorites and comets, Pollack and Bodenheimer predict the $\mathrm{N} / \mathrm{C}$ ratio in the 
TABLE I

Comparison of Prediction on Elemental Abundances Compared to Solar Value with OUR RESUlts

\begin{tabular}{ll}
\hline Pollack and Bodenheimer (1988) & \multicolumn{1}{c}{ Our results } \\
\hline C 30 times solar (measured) & 30 times solar (assumed) \\
N 10-20 times solar & $1-150$ \\
S $>50-100$ times solar & $>100$ times solar \\
$>100$ times solar & $>$ solar \\
\hline
\end{tabular}

envelopes of the giant planets to be subsolar as well. Table I shows a summary of the predictions of Pollack and Bodenheimer (1989, Pollack, private communication), together with our results. From our calculations the sulfur mixing ratio is best determined, and agrees with the formation model discussed here. Nitrogen is definitely much less abundant than sulfur; unfortunately we cannot determine whether it is less abundant than carbon, as predicted by the models. Similarly, we cannot give any clues to the enhancement in the mixing ratio of water (or more precisely, oxygen).

\section{ATmospheric Dynamics AND Cloud Microphysics}

\section{Global Distribution of Ammonia Gas: \\ Vertical Mixing versus Supersaturation Model}

$i$. Vertical mixing. If ammonia gas is distributed uniformly in the atmosphere by vertical mixing, as suggested in Section 3Bi), the time scale for mixing should be short compared to that for sublimation in the subsaturated air and condensation in the supersaturated air. The time constant to reach diffusive equilibrium in an atmosphere is approximately $H^{2} / K$, with $H$ the scale height $(\sim 100 \mathrm{~km})$, and $K$ the eddy diffusion coefficient. The time for sublimation depends upon the sedimentation time of the particle: $10(100)-\mu \mathrm{m}$ sized particles fall through a cloud of thickness $50-80 \mathrm{~km}$ within approximately 80 (1) days. As soon as it drops below the cloud base into the subsaturated air, the particle will sublime immediately. Carlson et al. (1988) expect particle sizes of $\sim 1000 \mu \mathrm{m}$ in the $\mathrm{NH}_{4} \mathrm{SH}$ cloud; according to their calculations such particles will form within $\sim 10^{4} \mathrm{sec}$. If precipitation is important in this cloud, particle sizes will likely be somewhat smaller, with a lower limit of $\sim 10 \mu \mathrm{m}$. Hence, if the ammonia gas is distributed uniformly in the atmosphere by vertical mixing processes, the eddy diffusion coefficient needs to be large considering the sedimentation times: $\sim 10^{7}\left(10^{9}\right) \mathrm{cm}^{2} \mathrm{sec}^{-1}$ for $10(100)-\mu \mathrm{m}$ sized particles, and even larger if the particles are as large as $1000 \mu \mathrm{m}$. In addition, one needs to consider condensation in the supersaturated air, which, through the mixing is filled with condensation nuclei. When we include this effect, the eddy diffusion coefficient needs to be raised by a few orders of magnitude: $K>10^{10} \mathrm{~cm}^{2} \mathrm{sec}^{-1}$.

Upper limits to the eddy diffusion coefficient can be obtained from the fact that ortho-para hydrogen is observed to be in equilibrium at the 1-bar level (Conrath and Gierasch 1984). Since it takes about $10^{8} \mathrm{sec}$ to equilibrate hydrogen, the eddy diffusion coefficient $K \leqslant 10^{5} \mathrm{~cm}^{2} \mathrm{sec}^{-1}$ at the 1-bar level. Although this limit is at slightly higher altitudes than the region we probe at centimeter wavelengths, we think it is unlikely that an increase in the eddy diffusion coefficient by many orders of magnitude will happen below the 1-bar level. Moreover, it seems unlikely that such a large eddy diffusion coefficient is confined to a limited region in the atmosphere: $\sim 150<T$ $<\sim 250 \mathrm{~K}$. The precise levels should also vary slightly with latitude on the planet to accommodate the spatially resolved radio images.

ii. Supersaturation. The number density in the $\mathrm{NH}_{4} \mathrm{SH}$ cloud layer, when both $\mathrm{H}_{2} \mathrm{O}$ and $\mathrm{H}_{2} \mathrm{~S}$ are enhanced in mixing ratio by a factor of 500 and $\mathrm{NH}_{3}$ by 15 , is typically a few hundred per cubic centimeter at the cloud base (assuming 100- $\mu \mathrm{m}$ particles), and decreases exponentially to below 0.1 $\mathrm{cm}^{-3}$ at temperatures below 200-240 K. Since the reaction $\mathrm{H}_{2} \mathrm{~S}+\mathrm{NH}_{3} \rightarrow \mathrm{NH}_{4} \mathrm{SH}$ is 
heterogeneous, it requires the presence of solid surfaces (e.g., aerosols). It is therefore plausible that no $\mathrm{NH}_{4} \mathrm{SH}$ is formed at $T<220-240 \mathrm{~K}$, due to the lack of surfaces or condensation nuclei (aerosols or $\mathrm{NH}_{4} \mathrm{SH}$ particles) at these levels in the atmosphere. As discussed above, the sedimentation time for 10 - to $1000-\mu \mathrm{m}$ particles is so much shorter than the time to redistribute particles throughout the atmosphere $\left(K \leqslant 10^{5}\right.$ $\mathrm{cm}^{2} \mathrm{sec}^{-1}$ at 1 bar) that it is plausible that the air above the 220-240 K level could have been cleared of condensation nuclei.

When we compare the vertical mixing and supersaturation models, we think that the latter model is more realistic than the mixing model. In addition, with the supersaturation model one can explain the equator-to-pole gradient observed in the radio images in a natural way (Section 4 and below).

iii. Latitudinal variation in the ammonia mixing ratio. As shown in Section 4, there is a strong gradient from the equator to the pole in the radio brightness temperature. This could be a real variation in temperature due to, e.g., a latitudinal variation in the internal heat source, but the likelihood of a phenomenon producing a temperature gradient as large as observed is remote. However, Uranus does not behave in the usual "normal" manner that the other outer planets do. The variation in brightness temperature more likely reflects a variation in the ammonia mixing ratio, such that the pole is depleted in ammonia gas relative to the equator (but both values relative to solar). This is similar to what was observed on Jupiter (de Pater 1986), and which was attributed to gas descending in the atmosphere in the warmer belt regions and rising in the cooler zones.

It was mentioned in Section 4 that the equatorial temperature would match calculations for an atmosphere in which ammonia gas is supersaturated at levels in the atmosphere where $T<241 \mathrm{~K}$ and the mixing ratio is $2 \times 10^{-6}$; at midlatitudes this level is near $220 \mathrm{~K}$ where the ammonia mixing ratio is $1-2 \times 10^{-7}$. This hints at a general upwelling at midlatitudes, with subsidence in the equatorial region (and at higher latitudes), so condensation nuclei could generally be found at higher altitudes at midlatitudes than in the equatorial region. This atmospheric circulation supports the meridional flow model as derived from Voyager observations by Flasar et al. (1987).

The polar region appears to have an ammonia mixing ratio slightly below that at midlatitudes, down to levels in the atmosphere where the temperature is approximately $280 \mathrm{~K}$. This hints at strong downdrafts of "dry" air (e.g., air from which $\mathrm{NH}_{3}$ has been removed by condensation, in either the $\mathrm{NH}_{3}$ ice cloud or the $\mathrm{NH}_{4} \mathrm{SH}$ cloud at midlatitudes) down to deep levels in Uranus' atmosphere.

\section{CONCLUSIONS}

We have investigated Uranus' radio spectrum as well as the pole-equator gradient in radio brightness temperature with help of model atmosphere calculations using the radiative transfer code developed by de Pater and Massie (1985) and a program which calculates the thermal structure of the atmosphere as developed by Romani (1986). In contrast to thermochemical equilibrium models (Section 3A), Uranus' spectrum can be matched only if there is a low ammonia mixing ratio distributed uniformly over a large range in altitude. The mixing ratio needs to be at least equal to or larger than the solar value in Uranus' deep atmosphere (Sections 3C and 5B). The decrease in ammonia mixing ratio is caused primarily by the formation of an $\mathrm{NH}_{4} \mathrm{SH}$ cloud, although a fair fraction $(\sim 65 \%)$ will get lost in the solution cloud if water is enhanced by a factor of $>500$ above the solar value. Regardless of the water mixing ratio, the $\mathrm{NH}_{4} \mathrm{SH}$ cloud must form at very deep levels in the atmosphere to deplete $\mathrm{NH}_{3}$ gas significantly at deep tropospheric levels. This is only possible if the $\mathrm{H}_{2} \mathrm{~S}$ mixing ratio (or S) is at least a hundred times the solar value. In addition, the $\mathrm{S} / \mathrm{N}$ ratio should be 
at least five times the solar value if $\mathrm{H}_{2} \mathrm{O}$ is enhanced by a factor $<100$ compared to the solar value; the $\mathrm{S} / \mathrm{N}$ ratio should be $>3$ if $\mathrm{H}_{2} \mathrm{O}$ is enhanced by $\geqslant 500$ over the solar value. These findings on mixing ratios are generally in agreement with the models on planetary formation by Pollack and Bodenheimer (1989).

The uniformity of the ammonia mixing ratio with altitude can best be explained in terms of the supersaturation model: at levels in the atmosphere where $T \leqslant 220-240$ $\mathrm{K}$, where the $\mathrm{NH}_{3}$ mixing ratio is a few times $10^{-7}$, no $\mathrm{NH}_{4} \mathrm{SH}$ is formed despite the fact that the product of the partial pressures of $\mathrm{H}_{2} \mathrm{~S}$ and $\mathrm{NH}_{3}$ exceeds the equilibrium constant. Since the reaction $\mathrm{H}_{2} \mathrm{~S}+\mathrm{NH}_{3} \rightarrow$ $\mathrm{NH}_{4} \mathrm{SH}$ only proceeds on solid surfaces, such as aerosols, this implies that the region in the atmosphere above where the temperature is $220-240 \mathrm{~K}$ is essentially clear of condensation nuclei. This is not unreasonable, considering the fact that the sedimentation time for 10- to $1000-\mu \mathrm{m}$ particles is much shorter than the time required for vertical mixing $\left(K \leqslant 10^{5} \mathrm{~cm}^{2}\right.$ $\left.\sec ^{-1}\right)$.

The pole-equator gradient in Uranus' radio brightness temperature can be explained best by assuming a latitude-dependent variation in the ammonia mixing ratio. In the equatorial regions, we need an $\mathrm{NH}_{3}$ mixing ratio about $2 \times 10^{-6}$ at altitudes where the temperature is $145<T<240 \mathrm{~K}$ to explain the brightness temperature observed at both 6 and $2 \mathrm{~cm}$; at midlatitudes we need roughly $10^{-7}$ at $T<220 \mathrm{~K}$, and in the polar region we need $\sim 10^{-7}$ down to 280 $K$. These numbers, coupled to the saturation model, imply a general upwelling at midlatitudes, with subsidence in the equatorial region. In the polar regions we need fast downdrafts of dry air (air from which $\mathrm{NH}_{3}$ gas has condensed out, in either the $\mathrm{NH}_{3}$ ice cloud or the $\mathrm{NH}_{4} \mathrm{SH}$ cloud at midlatitudes) to explain the low ammonia mixing ratio to such deep levels in the atmosphere.

We further note that the ammonia mixing ratio is symmetric about the pole at deep levels in the atmosphere (probed at $6 \mathrm{~cm}$ ), but is symmetric about a point between the pole and the subsolar point at higher levels (probed at $2 \mathrm{~cm}$ ). The general zonal appearance varies on time scales from maybe weeks (?) to years.

\section{FUTURE WORK}

Hubbard (1988) compared our atmospheric models with the $J_{4}$ value of Uranus' gravity field, as recently published by French et al. (1988). This low a $J_{4}$ value cannot accommodate models with the enrichment factors needed to explain the radio spectrum; in fact, one has even trouble fitting models which have a solar composition envelope with only $\mathrm{CH}_{4}$ enhanced by a factor of 30. Since French et al. (1988) assumed all higher order $J$-moments to be equal to zero, the actual $J_{4}$ value may be different than the published number. We hope this problem will be investigated in more detail in the future.

\section{ACKNOWLEDGMENTS}

We thank the many people with whom we had fruitful discussions during our research, in particular W. B. Hubbard, M. Podolak, and J. B. Pollack. I.dP. acknowledges support by NSF Grant AST-8514896 to the University of California and the Alfred P. Sloan Foundation; S.K.A. acknowledges support received from NASA Grant NSG-7404; P.N.R. acknowledges support from the NAS/NRC Resident Research program at NASA/GSFC.

\section{REFERENCES}

ATREYA, S. K. 1986. Atmospheres and lonospheres of the Outer Planets and Their Satellites. New York.

AtreyA, S. K., AND P. N. Romani 1985. Photochemistry and clouds of Jupiter, Saturn and Uranus. In Planetary Meteorology (G. E. Hunt, Ed.), pp. 17 68. Cambridge Univ. Press, Cambridge.

Atreya, S. K., B. R. Sandel, and P. N. Romani 1988. Photochemistry and vertical mixing. In Uranus (J. T. Bergstrahl, Ed.). Univ. of Arizona Press, Tucson.

Bragin, J., M. Diem, D. Guthals, and S. Chang 1977. The vibrational spectrum and lattice dynamics of polycrystalline ammonium hydrosulfide. $J$. Chem. Phys. 67, 1247-1255. 
Briggs, F. H., AND B. H. ANDREw 1980. Microwave radiometry and interferometry of Uranus. Icarus 41, 269-277.

Carlson, B. E., M. J. Prather, and W. B. Rossow 1987. The role of aqueous chemistry in determining the composition and cloud structure of the upper trophosphere on Uranus. Astrophys. J. Lett. 321, L97-L101.

Carlson, B. E., W. B. Rossow, and G. S. Orton 1988. Cloud microphysics of the giant planets. $J$. Atmos. Sci. 45, 2066-2081.

Castellan, G. W. 1971. Physical Chemistry. Addison-Wesley, Reading, MA.

Clifford, I. L., AND E. Hunter 1933. The system ammonia-water at temperatures up to $150^{\circ} \mathrm{C}$ and at pressures up to twenty atmospheres. J. Phys. Chem. 37, 101-118.

Conrath, B., D. Gautier, R. Hannel, G. Lindal, AND A. MARTEN 1987. The helium mixing ratio of Uranus from Voyager measurements. J. Geophys. Res. 92, 15003-15010.

Conrath, B. J., and P. J. Gierasch 1984. Global variation of the para hydrogen fraction in Jupiter's atmosphere and implications for dynamics on the outer planets. Icarus 57, 184-204.

DE PATER, I. 1986. Jupiter's zone-belt structure at radio wavelengths. II. Comparison of observations with model atmosphere calculations. Icarus 68, 344-365.

de Pater, I., ANd S. Gulkis 1988. VLA observations of Uranus at $1.3-20 \mathrm{~cm}$. Icarus 75, 306-323.

de Pater, I., ANd S. T. Massie 1985. Models of the millimeter-centimeter spectra of the giant planets. Icarus 62, 143-171.

Fegley, B., JR., AND R. G. Prinn 1986. Chemical models of the deep atmosphere of Uranus. Astrophys. J. 307, 852-865.

Flasar, F. M., B. J. Conrath, P. J. Gierasch, and J. A. Piraglia 1987. Voyager infrared observations of Uranus' atmosphere: thermal structure and dynamics. J. Geophys. Res. 92, 15,011-15,018.

French, R. G., J. L. Elliot, L. M. French, J. A. Kangas, K. J. Meech, M. E. Ressler, M. W. Buie, J. A. Frogel, J. B. Holberg, J. J. FuensaLIDA, AND M. JoY 1988. Uranian ring orbits from Earth based and Voyager occultation observations. Icarus 73, 349-378.

Goodman, G. C. 1969. Models of Jupiter's Atmosphere. Ph.D. dissertation, University of Illinois, Urbana.

Gulkis, S., AND I. DE Pater 1984. A review of the millimeter and centimeter observations of Uranus. In Uranus and Neptune: Proc. of a workshop held in Pasadena, CA, Feb. 6-8, 1984, pp. 225-262. NASA Conference Publ. 2330.

Gulkis, S., M. J. Janssen, and E. T. Olsen 1978. Evidence for the depletion of ammonia in the Uranus atmosphere. Icarus 34, 10-19.

Gulkis, S., E. T. Olsen, M. J. Klein, AND T. J.
Thompson 1983. Uranus: Variability of the microwave spectrum. Science 221, 453-455.

HubBard, W. B. 1988. Private communication. International Critical Tables 1930. McGraw-Hill.

ISAMBERT, M. 1881. Etude de la vapeur de bisulfhydrate d'ammoniaque. Compt. Rend. 92, 919-922.

ISAMBERT, M. 1882. Sur le bisulfhydrate et al cyanhydrate d'ammoniaque. Compt. Rend. 94, 958-960.

JAFFe, W. J., G. L. Berge, T. OWEN, AND J. Caldwell 1984. Uranus: Microwave images. Science 225, 619-621.

Klein, M. J., And J. A. Turegano 1978. Evidence of an increase in the microwave brightness temperature of Uranus, Astrophys. J. Lett. 224, L,31-L34.

LASKeR, B. M. 1963. Wet adiabatic model atmospheres for Jupiter. Astrophys. J. 138, 709-719.

LEYKo, J. 1964. Equilibrium studies on the $\mathrm{H}_{2} \mathrm{~S}-\mathrm{NH}_{3}-$ $\mathrm{H}_{2} \mathrm{O}$ system. I. Bull. Acad. Polon. Sci. Ser. Chim. 12, 275-276.

LEWIS, J. S. 1969. The clouds of Jupiter and the $\mathrm{NH}_{3}$ $\mathrm{H}_{2} \mathrm{O}$ and $\mathrm{NH}_{3}-\mathrm{H}_{2} \mathrm{~S}$ systems. Icarus $10,365-378$.

Lewis, J. S., AND R. G. Prinn 1980. Kinetic inhibition of $\mathrm{CO}$ and $\mathrm{N}_{2}$ reduction in the solar nebula. Astrophys. J. 238, 357-364.

Lindal, G. F., J. R. Lyons, D. N. SweEtnam, V. R. Eshelman, D. P. Hinson, AND G. L. Tyler 1987. The atmosphere of Uranus: Results of radio occultation measurements with Voyager 2. J. Geophys. Res. 92, 14,987-15,002.

Magnusson J. P. 1907. Equilibrium between ammonia and hydrogen sulphide. J. Phys. Chem. 11, 2146.

Massie, S. T., AND D. M. Hunten 1982. Conversion of para and ortho hydrogen in the Jovian planets. Icarus 49, 213-226.

Pollack, J. B., AND P. Bodenheimer 1989. Theories of the origin and evolution of the giant planets. In Origin and Evolution of Atmospheres (S. K. Atreya, J. B. Pollack and M. S. Matthews, Eds.), pp. 564602.

PrinN, R. G., AND J. S. Lewis 1973. Uranus atmosphere: Structure and composition. Astrophys. $J$. 179, 333-342.

Romani, P. N. 1986. Ph.D. thesis, University of Michigan, Ann Arbor.

Romani, P. N., and S. K. Atreya 1988. Methane photochemistry and haze production on Neptune. Icarus 74, 424-445.

StefFes, P. G., AND J. M. Jenkins 1987. Laboratory measurements of the microwave opacity of gaseous ammonia $\left(\mathrm{NH}_{3}\right)$ under simulated conditions for the Jovian atmosphere. Icarus 72, 35-47.

Trafton, L. M. 1976. The aerosol distribution in Jupiter's atmosphere: Interpretation of the hydrogen spectrum. Astrophys. J. 207, 1007-1024.

Wagman, D. D., W. H. Evens, I. Halow, V. B. Parker, S. M. Bailey, ANd R. H. Schumm 1965. Selected values of chemical thermodynamic proper- 
ties. Part 1. Tables for the first twenty-three elements in the standard order of arrangement. $\mathrm{Na}$ tional Bureau of Standards Technical Note 270-1.

WALKER, J., AND J. S. LuMSDEN 1897. Dissociationpressure of hydrosulphides. J. Chem. Soc. London, Trans. 71, 428-440.
Weidenschilling, S. J., ANd J. S. Lewis 1973. Atmospheric and cloud structures of the Jovian planets. Icarus 20, 465-476.

Wilson, J. A. 1925. The total and partial vapor pressures of aqueous ammonia solutions. Univ. Ill. Eng. Exp. Sta. Bull. 146. 